JOURNAL OF THE

AMERICAN MATHEMATICAL SOCIETY

Volume 16, Number 2, Pages 303-329

S 0894-0347(02)00417-4

Article electronically published on December 3, 2002

\title{
RELATIONS IN THE COHOMOLOGY RING OF THE MODULI SPACE OF RANK 2 HIGGS BUNDLES
}

\author{
TAMÁS HAUSEL AND MICHAEL THADDEUS
}

Let $C$ be a smooth complex projective curve of genus $g$. By a Higgs bundle on $C$ we shall mean a pair $(E, \phi)$ consisting of a holomorphic vector bundle $E$ on $C$ and a section $\phi \in H^{0}(C$, End $E \otimes K(n p))$, where $n \geq 0$ is fixed and $p \in C$ is a basepoint. With the appropriate notion of stability, there exists a quasi-projective moduli space $\mathcal{H}_{n}$ of stable Higgs bundles of fixed rank and degree. The aim of this paper is to characterize the rational cohomology ring of $\mathcal{H}_{n}$ when the rank is 2 and the degree is odd.

In fact, we have given a complete set of generators for this ring in another paper [14. So it is now a question of finding the relations between these generators. Even though this is a natural companion to the generation problem, the ideas and methods with which it is studied have quite a different flavor. In particular, there is much more explicit calculation.

What makes the rank 2 case tractable is that the number of generators is manageable: just $\varepsilon_{1}, \ldots, \varepsilon_{2 g} \in H^{1}, \alpha \in H^{2}, \psi_{1}, \ldots, \psi_{2 g} \in H^{3}$, and $\beta \in H^{4}$. In arbitrary rank, the number of generators is quite large, and finding all the relations seems out of reach at the moment. At any rate, it has not even been done for the compact moduli space $\mathcal{N}$ of stable bundles which lies inside $\mathcal{H}_{n}$.

Now in rank 2 the relations in the cohomology ring of $\mathcal{N}$ have been calculated by several authors [4, 16, 25, [33], and of course our ideal of relations must contain theirs. However, the answer to our problem is actually more explicit in that the relations are given by a simple closed formula, rather than by a recursion in the genus $g$.

Yet in another sense our answer is more complicated. Although our relations are completely explicit, there are very many of them, and there is no escaping this. The so-called "invariant part" of $H^{*}(\mathcal{N})$, for example, requires only 3 relations to generate all the others multiplicatively, while the corresponding number for $H^{*}\left(\mathcal{H}_{n}\right)$ grows quadratically in $g$.

Within this large crowd, some old friends stand out. For example, $\beta^{g}$, conjectured by Newstead [23] in 1972 to be a relation on $\mathcal{N}$ and shown to be one much later [18, 29, remains a relation on $\mathcal{H}_{0}$, though not on all of the $\mathcal{H}_{n}$.

Another familiar part of the story is a proposition proved in $\sqrt{10}$, stating that, if $\rho$ is a relation on $\mathcal{H}_{0}$ at genus $g-1$, then $\psi_{j} \psi_{j+g} \rho$ is a relation on $\mathcal{H}_{0}$ at genus g. Results like this have appeared several times before in the subject [22, 29]

Received by the editors June 10, 2002.

2000 Mathematics Subject Classification. Primary 14H60; Secondary 14D20, 14H81, 32Q55, $58 \mathrm{D} 27$.

The first author was supported by NSF grant DMS-97-29992.

The second author was supported by NSF grant DMS-98-08529. 
31. They rely strongly on the interpretation of the moduli space in terms of flat connections (or more precisely, connections of constant central curvature). Indeed, this marks the sole point where this interpretation, otherwise banished in favor of Higgs bundles, is briefly recalled.

The contents of the paper may be summarized as follows. Section 1 recalls the existing results we need, notably the generation theorem. Section 2 states the main result of the paper, which gives an explicit basis $\rho_{r, s, t}^{c}$ for the ideal of relations in rank 2. Section 3 then reviews some basic facts about equivariant cohomology, which is the main technical tool. Section 4 classifies the components of the fixedpoint set of the $\mathbb{C}^{\times}$-action on $\mathcal{H}_{n}$ in whose equivariant cohomology we will work. These are closely related to the symmetric products of $C$, and so $\$ 5$ reviews some relevant facts about symmetric products of curves. Using these facts, $\sqrt{6}$ computes the restrictions of the generators to each component of the fixed-point set. This is crucial information if a polynomial in these classes is to be shown to be a relation, since a theorem of Kirwan (3.1) (iii) implies that a polynomial in the generators is an equivariant relation if and only if its restriction to every component of the fixed-point set vanishes.

Section 7 defines some polynomials $\xi_{r, s}^{k}$ in $\alpha, \beta, \gamma$, by a recursive process. Then in $\$ 8$ we finally compute our first equivariant relations, by showing that certain combinations of the $\xi_{r, s}^{k}$ vanish when restricted to the fixed-point set. These relations are expressed in terms of the explicit polynomials $\rho_{r, s, t}^{c}$ by a purely algebraic argument, given in 9. The proposition discussed before, about multiplication by $\gamma$, is proved next, in $\$ 10$ it provides many new relations which are divisible by $\gamma$.

Up to this point, all the relations we have considered are polynomials in $\alpha, \beta, \gamma$, and thus in particular invariant under the action of the symplectic group $\Gamma=$ $\operatorname{Sp}(2 g, \mathbb{Z}) ; 11$ remedies this situation, showing how the invariant relations may be used to deduce many more noninvariant relations. In fact, the relations on $\mathcal{H}_{n}$ at genus $g$ divisible by $\psi_{1} \psi_{2} \cdots \psi_{k}$ turn out to be exactly the invariant relations on $\mathcal{H}_{n+k}$ at genus $g-k$. Thus, even if one is interested only in the space $\mathcal{H}_{0}$ parametrizing connections of constant central curvature, one needs to understand the cohomology of $\mathcal{H}_{n}$ for $n>0$.

Finally, 12 wraps up the proof of the main theorem, by showing that the number of relations we already have in hand equals the total number.

The story of how these relations were conjectured and proved is worth telling briefly. It was clear from the outset that Kirwan's theorem would be an invaluable tool; that it could be used, as in $\$ 8$, to decide whether a polynomial in the equivariant generators is a relation; and that this was, in principle, completely algorithmic. The first author was able to implement this algorithm in the computer software package Macaulay 2 [9] and crank out the equivariant relations for $g \leq 7$. Their restrictions to ordinary cohomology showed some discernible patterns, so after two weeks in Oberwolfach, we were able to guess all of the ordinary relations for general $g$, in roughly the form $\rho_{r, s, t}^{c}$ given in (2.3). It remained only to guess their equivariant extensions, that is, the relations in the equivariant cohomology, which has one extra generator. Guessing these equivariant extensions took the better part of two years.

More precisely, we were never able to guess a closed formula or generating function for the equivariant extensions of the $\rho_{r, s, t}^{c}$ themselves, even though we knew them in genus $\leq 7$. If we could have, this would have been a much shorter paper. 
We hope that someone will guess these extensions some day; what we actually did, though, was to find rather different equivariant relations (8.5), and then show by several artful maneuvers, including the proposition mentioned above, that these imply the relations $\rho_{r, s, t}^{c}$ we originally found.

Some other papers of the authors have explored different aspects of the theory of Higgs bundles: for example, the compactification of the moduli space [11, its intersection numbers in the compactly supported cohomology [12, and the upward and downward flows from the components of the fixed-point set 32. Some relationships between these topics and the contents of this paper are sketched in the last section, \$13.

Notation and conventions. Throughout the paper, $C$ denotes the smooth projective curve of genus $g$ over which we work. Its cohomology has the usual generators $e_{1}, \ldots, e_{2 g} \in H^{1}$, and $\sigma=e_{j} e_{j+g} \in H^{2}$. The $m$ th symmetric product of $C$ is denoted $C_{m}$, and the Jacobian of degree $d$ line bundles on $C$ is denoted $\operatorname{Jac}^{d} C$. The letters $\mathcal{N}, \mathcal{H}_{n}$, and $\mathcal{M}_{n}$ denote moduli spaces over $C$, respectively, of stable bundles $E$ having $\Lambda^{2} E$ isomorphic to a fixed line bundle $\Xi$, of Higgs bundles with values in $K(n)=K \otimes \mathcal{O}(n p)$, and Higgs bundles $(E, \phi)$ with values in $K(n)$ having $\Lambda^{2} E \cong \Xi$ and $\operatorname{tr} \phi=0$. Groups are denoted $T=\mathbb{C}^{\times}, \Gamma=\operatorname{Sp}(2 g, \mathbb{Z})$, and $\Sigma=\mathbb{Z}_{2}^{2 g}$.

We use the term total degree to mean half the ordinary degree of a cohomology class. The notation ()$_{d}$ means the part of a cohomology class in total degree $d$. All cohomology is with rational coefficients unless otherwise stated.

We do not assume $g \geq 2$ : the moduli spaces $\mathcal{M}_{0}, \mathcal{H}_{0}$ and $\mathcal{N}$ are trivial or empty if $g=0$ or 1 , but $\mathcal{M}_{n}$ and $\mathcal{H}_{n}$ for $n>0$ are not so trivial, and they play an important role even for understanding $g \geq 2$.

\section{Preliminaries and Review}

Let $C$ be a smooth complex projective curve of genus $g$, and let $p \in C$ be a distinguished point. For $n \geq 0$, denote by $K(n)$ the line bundle $K \otimes \mathcal{O}(n p)$, where $K$ is the canonical bundle. A Higgs bundle with values in $K(n)$ is a pair $(E, \phi)$ consisting of a holomorphic vector bundle $E$ over $C$ and a Higgs field $\phi \in$ $H^{0}($ End $E \otimes K(n))$. It is stable if for all proper subbundles $F \subset E$ such that $\phi(F) \subset F \otimes K(n), \operatorname{deg} F / \operatorname{rk} F<\operatorname{deg} E / \operatorname{rk} E$.

The existing results we shall need to recall are few and, except for the authors' result stated as (1.4) below, have been known for some time. They can be summarized as follows.

First, there are some elementary facts about stable Higgs bundles [14, 4.2,4.3].

(1.1) If $X$ parametrizes two families $(\mathbf{E}, \boldsymbol{\Phi})$ and $\left(\mathbf{E}^{\prime}, \boldsymbol{\Phi}^{\prime}\right)$ of stable Higgs bundles, and $\left(\mathbf{E}_{x}, \boldsymbol{\Phi}_{x}\right) \cong\left(\mathbf{E}_{x}^{\prime}, \boldsymbol{\Phi}_{x}^{\prime}\right)$ for all $x \in X$, then $\mathbf{E}^{\prime}=\mathbf{E} \otimes L$ for some line bundle $L$ over $X$, and $\boldsymbol{\Phi}^{\prime}=\boldsymbol{\Phi}$. Moreover, if an action of $\mathbb{C}^{\times}$on $X$ lifts to $\mathbf{E}$ and $\mathbf{E}^{\prime}$ preserving the Higgs fields, then it lifts to $L$ also so that $\mathbf{E}^{\prime}=\mathbf{E} \otimes L$ equivariantly.

Next, there is a moduli space, constructed by Simpson [27] and Nitsure [24].

(1.2) There exists a moduli space $\mathcal{H}_{n}$ of stable Higgs bundles of rank 2 and degree 1 with values in $K(n)$, which is a smooth quasi-projective variety. It admits a universal family $(\mathbf{E}, \boldsymbol{\Phi})$, and the $\mathbb{C}^{\times}$-action on $\mathcal{H}_{n}$ given by $\lambda(E \phi)=(E, \lambda \phi)$ lifts to this family. 
The following alternative interpretation of $\mathcal{H}_{0}$, due to Corlette [5], Donaldson [6], Hitchin [15], and Simpson [27], will only be used in \$10.

(1.3) For $n=0$, the moduli space $\mathcal{H}_{0}$ is diffeomorphic to the moduli space $\mathcal{H}$ of $\mathrm{GL}(2, \mathbb{C})$-connections of constant central curvature $i \omega I$, where $\omega$ is a volume form on $C$. That is, $\mathcal{H}_{0} \simeq \mu^{-1}(-I) / \mathrm{GL}(2, \mathbb{C})$, where $\mu: \operatorname{GL}(2, \mathbb{C})^{2 g} \rightarrow \mathrm{GL}(2, \mathbb{C})$ is given by $\mu\left(A_{j}, B_{j}\right)=\prod_{j=1}^{g} A_{j} B_{j} A_{j}^{-1} B_{j}^{-1}$, and $\mathrm{GL}(2, \mathbb{C})$ acts on $\mu^{-1}(-I)$ by simultaneous conjugation. The natural determinant maps and universal families coincide under this diffeomorphism.

Let $\Xi$ be a fixed holomorphic line bundle over $C$ of degree 1 , and let $\mathcal{M}_{n} \subset \mathcal{H}_{n}$ be the subspace consisting of those $(E, \phi) \in \mathcal{H}_{n}$ such that $\Lambda^{2} E \cong \Xi$ and $\operatorname{tr} \phi=0$. In the case $n=0$, this is the moduli space studied by Hitchin [15]. The discussion so far, and the previous paper of the authors [14, refer to $\mathcal{H}_{n}$, but the remainder of this paper will actually work with $\mathcal{M}_{n}$. This gives equivalent information for the following reason. The group $\Sigma=\mathbb{Z}_{2}^{2 g} \subset \mathrm{Jac} C$ of line bundles with structure group $\mathbb{Z}_{2}$ acts on $\mathcal{M}_{n}$ by tensor product, and indeed $\mathcal{H}_{n}=\left(\mathcal{M}_{n} \times T^{*} \operatorname{Jac} C\right) / \Sigma$. As seen in $\S 1$ of our previous paper [14], $H^{*}\left(\mathcal{H}_{n}\right)=H^{*}\left(\mathcal{M}_{n}\right)^{\Sigma} \otimes H^{*}(\operatorname{Jac} C)$ as rings. To describe $H^{*}\left(\mathcal{H}_{n}\right)$, therefore, it suffices to describe $H^{*}\left(\mathcal{M}_{n}\right)^{\Sigma}$. This will be the purpose of the paper. The part of $H^{*}\left(\mathcal{M}_{n}\right)$ not invariant under $\Sigma$ is ignored here, but it is completely described in a forthcoming work [32].

The main result of our paper on the generators [14] is the following.

(1.4) The rational cohomology ring $H^{*}\left(\mathcal{M}_{n}\right)^{\Sigma}$ is generated by the universal classes, that is, the Künneth components $\alpha_{2}, \beta_{2}$, and $\psi_{2, j}$ of $\bar{c}_{2}(\mathbb{P E})=\frac{1}{4} c_{2}($ End $\mathbf{E})$.

This result has been extended to higher rank Higgs bundles by Markman 21].

Following the conventions established by Newstead [23], we will let $\alpha=\frac{1}{2} \alpha_{2}$, $\beta=-\frac{1}{4} \beta_{2}$, and $\psi_{j}=\psi_{2, j}$ for $j=1, \ldots, 2 g$, so that

$$
c_{2}(\text { End } \mathbf{E})=2 \alpha \sigma-\beta+4 \sum_{j=1}^{g} \psi_{j} e_{j},
$$

where $e_{1}, \ldots e_{2 g}$ is the usual basis for $H^{1}(C)$, and $\sigma \in H^{2}(C)$ is the positive generator.

\section{Statement of the main Result}

Our task, then, is to give a complete set of relations between the generators $\alpha$, $\beta$, and $\psi_{1}, \ldots, \psi_{2 g}$. To do so, we must first say a little about the action of the symplectic group on $H^{*}\left(\mathcal{M}_{n}\right)^{\Sigma}$.

The group of orientation-preserving diffeomorphisms of $C$ acts on $H^{*}(C)$ by automorphisms, so it has the automorphism group of $H^{*}(C)$, namely $\Gamma=\operatorname{Sp}(2 g, \mathbb{Z})$, as a quotient [19, p. 178].

(2.1) There is a natural action of $\Gamma$ on $H^{*}\left(\mathcal{M}_{n}\right)^{\Sigma}$ which fixes $\alpha$ and $\beta$ but acts on the $\psi_{j}$ as the standard representation.

Proof. In the case of $\mathcal{M}_{0}$, this follows immediately from (1.3), but to extend it to $\mathcal{M}_{n}$ we describe another argument.

Let $f: C \rightarrow C$ be any orientation-preserving diffeomorphism. The complex structure typically is not preserved by $f$, so pulling it back induces a new complex structure $C^{\prime}$ on the same underlying surface. Since Teichmüller space, or the moduli 
space of curves, is connected, there is a path connecting $C$ to $C^{\prime}$. The construction of the moduli space $\mathcal{M}_{n}$, and of the universal pair $(\mathbf{E}, \boldsymbol{\Phi})$, can be carried out simultaneously over all the Riemann surfaces in this path. Hence by homotopy invariance there is a topological isomorphism $\mathbf{E} \cong \mathbf{E}^{\prime}$, where $\left(\mathbf{E}^{\prime}, \boldsymbol{\Phi}^{\prime}\right)$ is a universal pair on $C^{\prime}$. The homotopy class of the isomorphism depends only on the isotopy class of $f$.

On the other hand, if $\hat{f}: \mathcal{M}_{n}^{\prime} \rightarrow \mathcal{M}_{n}$ is the map of moduli spaces induced by $f: C^{\prime} \rightarrow C$, then $(\hat{f} \times f)^{*}(\mathbf{E}, \boldsymbol{\Phi})$ is a universal pair over $C^{\prime}$, and so by the uniqueness in (1.1),

$$
(\hat{f} \times f)^{*} \text { End } \mathbf{E} \cong \text { End } \mathbf{E}^{\prime} \cong \text { End } \mathbf{E} .
$$

Hence $(\hat{f} \times f)^{*} c_{2}($ End $\mathbf{E})=c_{2}($ End $\mathbf{E})$, so $\hat{f}^{*} \alpha=\alpha, \hat{f}^{*} \beta=\beta$, and $(\hat{f} \times f)^{*} \sum_{j} \psi_{j} e_{j}=$ $\sum_{j} \psi_{j} e_{j}$. The action of the diffeomorphism group on $H^{3}\left(\mathcal{M}_{n}\right)=\left\langle\psi_{j}\right\rangle$ is therefore dual to its action on $H^{1}(C)=\left\langle e_{j}\right\rangle$; this factors through the standard representation of $\Gamma$, which is self-dual. Moreover, by (1.4), the action of the diffeomorphism group on all of $H^{*}\left(\mathcal{M}_{n}\right)^{\Sigma}$ factors through $\Gamma$.

The exterior square of the standard representation of $\gamma$ has an invariant element, the symplectic form. So $\gamma=-2 \sum_{j=1}^{g} \psi_{j} \psi_{j+g} \in H^{6}\left(\mathcal{M}_{n}\right)$ is a $\Gamma$-invariant element. Since the powers of the symplectic form are the only invariant elements of exterior powers of the standard representation, we deduce the following from (1.4). $\gamma \in H^{6}$.

The $\Gamma$-invariant part of $H^{*}\left(\mathcal{M}_{n}\right)^{\Sigma}$ is generated by $\alpha \in H^{2}, \beta \in H^{4}$, and

Like the exterior square discussed above, the higher exterior powers of the standard representation of $\Gamma$ are reducible. Indeed, let $\Lambda^{k}(\psi)$ be the $k$ th exterior power of the standard representation, with basis $\psi_{1}, \ldots, \psi_{2 g}$. Define the primitive part $\Lambda_{0}^{k}(\psi)$ to be the kernel of the natural map $\Lambda^{k} \rightarrow \Lambda^{2 g+2-k}$ given by the wedge product with $\gamma^{g+1-k}$. The primitive part is complementary to $\gamma \Lambda^{k-2} \subset \Lambda^{k}$ and is an irreducible representation of $\Gamma$ : this is well known for $\operatorname{Sp}(2 g, \mathbb{C})$ and so remains true for the Zariski dense subgroup $\Gamma$. Being irreducible, it is generated by $\psi_{1} \cdots \psi_{k}$.

For any $g, n \geq 0$, let $I_{n}^{g}$ be the ideal within the polynomial ring $\mathbb{Q}[\alpha, \beta, \gamma]$ generated by $\gamma^{g+1}$ and the polynomials

$$
\rho_{r, s, t}^{c}=\sum_{i=0}^{\min (c, r, s)}(c-i) ! \frac{\alpha^{r-i}}{(r-i) !} \frac{\beta^{s-i}}{(s-i) !} \frac{(2 \gamma)^{t+i}}{i !},
$$

where $c=r+3 s+2 t-2 g+2-n$, for all $r, s, t \geq 0$ such that

$$
r+3 s+3 t>3 g-3+n \quad \text { and } \quad r+2 s+2 t \geq 2 g-2+n .
$$

The following is then the main result of the present paper.

$$
\begin{aligned}
& \text { As a } \Gamma \text {-algebra, } \\
& \qquad H^{*}\left(\mathcal{M}_{n}\right)^{\Sigma}=\bigoplus_{k=0}^{g} \Lambda_{0}^{k}(\psi) \otimes \mathbb{Q}[\alpha, \beta, \gamma] / I_{n+k}^{g-k} .
\end{aligned}
$$

The theorem enunciated in (1.2) of our previous paper [14] is the above in the case $n=0$. In that case the relation of lowest degree is $\rho_{1, g-1,0}^{g}=g \alpha \beta^{g-1}+$ $(g-1) \beta^{g-2}(2 \gamma)$. When $n=1$, there are two relations of lowest degree, one of which is $\rho_{0, g, 0}^{g+1}=(g+1) \beta^{g}$. When $n \geq 2$, the lowest degree in which a relation appears is 
$2(2 g-2+n)$. At least for $s \geq r$, the relations in this degree have the particularly simple form $\beta^{s-r}(\alpha \beta+2 \gamma)^{r}$.

\section{Equivariant COHOMOLOGY}

Our main tool for studying the ring structure of $H^{*}\left(\mathcal{M}_{n}\right)$ is equivariant cohomology, which we briefly review. For a more leisurely exposition see Atiyah-Bott [2, 3].

If a group - say a Lie group - acts on a topological space $M$, the homotopy quotient $M_{G}$ is defined as the associated bundle over the classifying space $B G$ with fiber $M$ :

$$
M_{G}=\frac{M \times E G}{G} .
$$

The equivariant cohomology of $M$ is defined to be simply the ordinary cohomology of $M_{G}$ :

$$
H_{G}^{*}(X)=H^{*}\left(X_{G}\right) .
$$

This is a module over $H^{*}(B G)$. Restricting to any fiber gives a natural map $H_{G}^{*}(M) \rightarrow H^{*}(M)$. Note also that if $G$ acts trivially on $M$, then $H_{G}^{*}(M)=$ $H^{*}(M) \otimes H^{*}(B G)$.

If the action of $G$ lifts to a linear action on a vector bundle $E$, then a vector bundle $E_{G}$ over $M_{G}$ can be defined in the obvious way. Thus a vector bundle equipped with such a lifting possesses well-defined equivariant characteristic classes lying in $H_{G}^{*}(M)$.

In our case the group acting is $T=\mathbb{C}^{\times}$, so that $B T=\mathbb{C P}^{\infty}$, and $H^{*}(B T)=\mathbb{Q}[u]$ where $u$ is a class of degree 2. Kirwan [17 18] proved the following fundamental results on $\mathbb{C}^{\times}$-actions.

(3.1) When $T=\mathbb{C}^{\times}$acts algebraically on a smooth quasi-projective $M$ so that $\lim _{\lambda \rightarrow 0} \lambda \cdot x$ exists for every $x$, then

(i) there is an additive isomorphism

$$
H^{i}(M) \cong \bigoplus_{d} H^{i+r_{d}}\left(F_{d}\right),
$$

where $\mathcal{F}=\bigcup_{d} F_{d}$ is the decomposition of the fixed-point set into components and $r_{d}$ is the dimension of the subbundle of $\left.T M\right|_{F_{d}}$ acted on with negative weight by $T$;

(ii) the Leray sequence of $M_{T} \rightarrow B T$ degenerates, so that $H_{T}^{*}(M) \cong H^{*}(M)$ $\otimes H^{*}(B T)$ additively, and the ring homomorphism $H_{T}^{*}(M) \rightarrow H^{*}(M)$ is surjective;

(iii) the restriction to the fixed-point set

$$
H_{T}^{*}(M) \longrightarrow H_{T}^{*}(\mathcal{F})=H^{*}(\mathcal{F})[u]
$$

is an injective ring homomorphism.

Statement (i) is perhaps most familiar in a symplectic context as stating that the moment map is a perfect Bott-Morse function. But statement (iii) is equally crucial for us since it respects the ring structure. Together with (ii), it will tell us that a polynomial in $\alpha, \beta$, and $\psi_{j}$ is a relation on $\mathcal{M}_{n}$ if and only if it is the value at $u=0$ of a polynomial in $\alpha, \beta, \psi_{j}$, and $u$ - the equivariant extension of the relation - whose restriction to $H_{G}^{*}\left(F_{d}\right)=H^{*}\left(F_{d}\right)[u]$ is a relation for each $d$. 


\section{Fixed points of THE CIRCLE ACtion}

We will study the action of $T=\mathbb{C}^{\times}$on $\mathcal{M}_{n}$ given simply by $\lambda \cdot(E, \phi)=(E, \lambda \phi)$. By (1.2) this lifts to the universal bundle, and hence the universal classes extend to equivariant classes, which by abuse of notation, we continue to denote $\alpha$, $\beta$, and $\psi_{j}$. They are canonical by the uniqueness in (1.1).

In light of (3.1), it is vital to determine the fixed-point set for this action. As discussed in (10.5) of our previous paper [14, this would be somewhat tricky in arbitrary rank. But now that we have restricted attention to rank 2 (and fixed determinant), it is not so hard. The lemma below is proved by Hitchin [15, 7.1] for $\mathcal{M}_{0}$, but his proof generalizes directly to $\mathcal{M}_{n}$.

(4.1)

The components of the fixed-point set $\mathcal{F}$ for the $T$-action on $\mathcal{M}_{n}$ are as follows.

(i) A component $F_{0}$ isomorphic to $\mathcal{N}$, the moduli space of stable bundles $E$ with $\Lambda^{2} E \cong \Xi$. It parametrizes Higgs bundles of the form $(E, 0)$.

(ii) Components $F_{1}, \ldots, F_{g+\left[\frac{n-1}{2}\right]}$ which are fibered products

$$
F_{d}=C_{2 g+n-1-2 d} \times \mathrm{Jac}^{2 d} C \mathrm{Jac}^{d} C,
$$

where the maps $C_{2 g+n-1-2 d} \rightarrow \mathrm{Jac}^{2 d} C$ and $\mathrm{Jac}^{d} C \rightarrow \mathrm{Jac}^{2 d} C$ are given by $D \mapsto K \Xi(n)(-D)$ and $L \mapsto L^{2}$, respectively. These parametrize Higgs bundles $(E, \phi)$ of the form $E=L \oplus \Xi L^{-1}, \phi=\left(\begin{array}{cc}0 & 0 \\ s & 0\end{array}\right)$, where $s$ is the section of $K L^{-2} \Xi(n)$ vanishing at $D$.

Hitchin went on to compute the cohomology of the fixed components of type (ii) as follows. By the Leray sequence

$$
H^{*}\left(F_{d}\right)=\bigoplus_{i \in \Sigma} H^{*}\left(C_{2 g+n-1-2 d}, \mathcal{L}_{i}\right),
$$

where the right-hand side consists of cohomology with local coefficients, and $\mathcal{L}_{i}$ runs over the flat line bundles with structure group $\mathbb{Z}_{2}$ pulled back from Jac ${ }^{2 d} C$. If $\mathcal{L}_{i}$ is the trivial bundle, this is simply the ordinary cohomology $H^{*}\left(C_{2 g+n-1-2 d}\right)$. Otherwise $H^{k}\left(C_{2 g+n-1-2 d}, \mathcal{L}_{i}\right)=\Lambda^{k} H^{1}\left(C, L_{i}\right)$ if $k=2 g+n-1-2 d$, and 0 if not. Here $L_{i}$ runs over the flat line bundles with structure group $\mathbb{Z}_{2}$ pulled back from $\mathrm{Jac}^{1} C$ to $C$ by the Abel-Jacobi map. Hitchin shows that for $L_{i}$ nontrivial, $H^{0}\left(C, L_{i}\right)=H^{2}\left(C, L_{i}\right)=0$, and $H^{1}\left(C, L_{i}\right)$ has dimension $2 g-2$.

The action of $\Sigma$ on $\mathcal{M}_{n}$ commutes with the $T$-action and induces the trivial action on $H^{*}(\mathcal{N})$ since it is generated by universal classes [23]. It acts on the remaining $F_{d}$ as the Galois group of the unbranched cover $F_{d} \rightarrow C_{2 g+n-1-2 d}$, and the splitting $(4.2)$ is exactly the decomposition of the cohomology into weight spaces.

Consequently, the $\Sigma$-invariant part of $H^{*}(\mathcal{F})$ is

$$
H^{*}(\mathcal{N}) \oplus \bigoplus_{d=1}^{g+\left[\frac{n-1}{2}\right]} H^{*}\left(C_{2 g+n-1-2 d}\right) .
$$

\section{Symmetric products of a CURVE}

The symmetric products of the curve $C$ thus enter into our considerations. So let us review some facts about the cohomology of such a symmetric product. Good references are the paper of Macdonald [20] or the book of Arbarello et al. [1]. 
In $C_{m} \times C$, there is a universal divisor $\Delta$ such that $\Delta \cap(\{D\} \times C)=D$. Write its Poincaré dual in terms of Künneth components as

$$
m \sigma+\eta+\sum_{j=1}^{2 g} \xi_{j} e_{j}
$$

where $\sigma$ and $e_{1}, \ldots, e_{2 g}$ are generators of $H^{2}(C)$ and $H^{1}(C)$, respectively, so that $\eta \in H^{2}\left(C_{m}\right)$ and $\xi_{1}, \ldots, \xi_{2 g} \in H^{1}\left(C_{m}\right)$. A theorem of Macdonald [20] asserts that the cohomology ring $H^{*}\left(C_{m}\right)$ is generated by $\eta$ and the $\xi_{j}$. It is convenient to introduce $\theta_{j}=\xi_{j} \xi_{j+g}$ and $\theta=\sum_{j=1}^{g} \theta_{j} \in H^{2}(C)$.

The group of orientation-preserving diffeomorphisms of $C$ acts on $C_{m} \times C$. It preserves $\Delta$ and hence the Künneth components of its Poincaré dual. Hence it leaves $\eta$ invariant. Moreover, its action on the linear span of the $\xi_{j}$, which is $H^{1}\left(C_{m}\right)$, is dual to its action on $H^{1}(C)$ and hence factors through the quotient $\Gamma=$ $\operatorname{Sp}(2 g, \mathbb{Z})$. There is therefore a surjective homomorphism of $\Gamma$-algebras $\Lambda^{*}(\xi)[\eta] \rightarrow$ $H^{*}\left(C_{m}\right)$. Here $\Lambda^{*}(\xi)$ denotes the exterior algebra of the standard $2 g$-dimensional representation of $\Gamma$, with basis vectors $\xi_{1}, \ldots, \xi_{2 g}$.

The class $\theta$ represents the symplectic form. So in terms of the primitive parts $\Lambda_{0}^{k}$ introduced in $\$ 2$ the surjective homomorphism above is better expressed as

$$
\bigoplus_{k=0}^{g} \Lambda_{0}^{k}(\xi) \otimes \mathbb{Q}[\eta, \theta] \longrightarrow H^{*}\left(C_{m}\right)
$$

In particular, the $\Gamma$-invariant part of $H^{*}\left(C_{m}\right)$ is generated by $\eta$ and $\theta$.

The following result on $H^{*}\left(C_{m}\right)$ will be of key importance for us. Note that we use the term total degree to mean half the ordinary degree of a cohomology class.

Let $l, m, p$, and $q$ be nonnegative integers. If $m-g+q \leq l$ and $g+p-q<l$, then

$$
\left(\frac{\eta^{p} \exp \theta}{(1+\eta)^{q}}\right)_{l}=0
$$

in $H^{*}\left(C_{m}\right)$, where the subscript $l$ denotes the part in total degree $l$.

Proof. Since the cup product is a homomorphism of $\Gamma$-modules, Poincaré duality holds for the $\Gamma$-invariant part. It therefore suffices to check that the product of this expression with any monomial in $\eta$ and $\theta$ evaluates to 0 on the fundamental class of $C_{m}$.

It follows from Macdonald's results [20] that any monomial $\eta^{v} \prod_{j} \xi_{j}^{w_{j}}$ of total degree $m$ evaluates on the fundamental class of $C_{m}$ to 1 if $w_{j}=w_{j+g} \leq 1$ for each $j \leq g$, and 0 otherwise. As pointed out by Zagier 30, this implies that for any formal power series $A(x)$ and $B(x)$,

$$
A(\eta) \exp (\theta B(\eta))\left[C_{m}\right]=\operatorname{Res}_{\eta=0} \frac{A(\eta)(1+\eta B(\eta))^{g} d \eta}{\eta^{m+1}} .
$$


We multiply our expression by the generating function $\exp (s \theta) /(1+t \eta)$ for the monomials in $\eta$ and $\theta$ and ask the coefficient of $s^{i} t^{j}$ to vanish whenever $i+j=m-l$ :

$$
\begin{aligned}
\underset{s^{i} t^{j}}{\operatorname{Coeff}} \frac{\eta^{p} \exp ((s+1) \theta)}{(1+\eta)^{q}(1+t \eta)}\left[C_{m}\right] & =\underset{s^{i} t^{j}}{\operatorname{Coeff}} \operatorname{Res}_{\eta=0} \frac{\eta^{p}(1+\eta+s \eta)^{g} d \eta}{(1+\eta)^{q}(1+t \eta) \eta^{m+1}} \\
& =\text { const. } \operatorname{Res}_{\eta=0} \frac{\eta^{p}(1+\eta)^{g-i} \eta^{i} \eta^{j} d \eta}{(1+\eta)^{q} \eta^{m+1}} \\
& =\text { const. } \operatorname{Res}_{\eta=0} \eta^{i+j+p-m-1}(1+\eta)^{g-i-q} d \eta .
\end{aligned}
$$

Now since $g-i-q \geq g-q-(m-l) \geq 0$ by hypothesis, the second factor is a polynomial of degree $g-i-q$. All terms therefore have degree at most

$(i+j+p-m-1)+(g-i-q) \leq p+(m-l)-m-1+g-q=p-l-1+g-q$, which is less than -1 by hypothesis.

\section{Restriction of the Universal ClAsses to THE FIXED-POINT SET}

In order to apply 3.1(iii), we need to know how the equivariant universal classes restrict to each component of the fixed-point set. The lowest component $F_{0}=\mathcal{N}$ is easy. The universal pair over $\mathcal{M} \times C$ restricts to a universal bundle over $\mathcal{N} \times C$, and the $T$-action restricts to a trivial action. So $\alpha, \beta$ and $\psi_{j}$ restrict to classes on $\mathcal{N}$ defined in a like manner and bearing the same names. The relations between these classes on $\mathcal{N}$ have been studied by many authors, notably Zagier [33] we will have occasion to use his results later.

The components $F_{d}$ for $d>0$ are handled by the following lemma.

(6.1) For $d>0$, the restrictions of the universal classes to $F_{d}$ are pulled back from the symmetric product $C_{2 g+n-1-2 d}$; indeed,

(1) $\left.\alpha\right|_{F_{d}}=(2 d-1)(\eta-u)+\theta$;

(2) $\left.\beta\right|_{F_{d}}=(\eta-u)^{2}$;

(3) $\left.\psi_{j}\right|_{F_{d}}=\frac{1}{2}(\eta-u) \xi_{j}$;

(4) $\left.\gamma\right|_{F_{d}}=-\frac{1}{2}(\eta-u)^{2} \theta$.

Proof. We first construct an equivariant universal family $(\mathbf{E}, \boldsymbol{\Phi})$ of Higgs bundles over $F_{d} \times C$. Since End $\mathbf{E}$ is unique as an equivariant bundle by (1.1), the universal classes must restrict to the Künneth components of its second Chern class.

Take the following three ingredients. First, take the line bundle $K \Xi(n)$ over $C$. Second, take the universal divisor $\Delta \subset C_{2 g+n-1-2 d} \times C$, or rather its associated line bundle $\mathcal{O}(\Delta)$. Third, take any Poincaré line bundle $\mathcal{L}$ over $\operatorname{Jac}^{d} C \times C$. Now pull all three back to $F_{d} \times C$. By the definition of the fibered product, $\mathcal{L}^{2}$ and $K \Xi(n)(-\Delta)$ are isomorphic when restricted to any fiber of the projection $F_{d} \times C \rightarrow F_{d}$. So by the push-pull formula, $\mathcal{L}^{2} K^{-1} \Xi^{-1}(-n)(\Delta)$ is the pull-back from $F_{d}$ of a line bundle, say $M$. There is then an element $s \in H^{0}\left(F_{d} \times C, M \mathcal{L}^{-2} K \Xi(n)\right)$ vanishing precisely on the inverse image of $\Delta$.

Let $\mathbf{E}=\mathcal{L} \oplus M \Xi \mathcal{L}^{-1}$, and let $\boldsymbol{\Phi} \in H^{0}($ End $\mathbf{E} \otimes K(n))$ be given by $\mathbf{\Phi}=\left(\begin{array}{cc}0 & 0 \\ s & 0\end{array}\right)$ with respect to the splitting. Then $(\mathbf{E}, \mathbf{\Phi})$ parametrizes the pairs of the form described in (4.1) (ii). It is hence a universal family. Moreover, if $T$ acts on the two factors with weights 1 and 0 , respectively, then it acts on $\boldsymbol{\Phi}$ by scalar multiplication. By (1.1), then, End $\mathbf{E}$ is equivariantly isomorphic to the restriction of its counterpart from $\mathcal{M}_{n} \times C$. 
Since $\mathbf{E}$ splits as a direct sum, $c_{2}($ End $\mathbf{E})=-\left(c_{1}\left(M \Xi \mathcal{L}^{-1}\right)-c_{1}(\mathcal{L})\right)^{2}=$ $-c_{1}\left(M \Xi \mathcal{L}^{-2}\right)^{2}=-c_{1}\left(K^{-1}(-n)(\Delta)\right)^{2}$. For this to be correct equivariantly, we must include the weights of the $T$-action, so the equivariant $c_{1}$ is the nonequivariant $c_{1}$ minus $u$. It is well known that

$$
c_{1}(\mathcal{O}(\Delta))=(2 g+n-1-2 d) \sigma+\eta+\sum_{j=1}^{2 g} \xi_{j} e_{j}:
$$

see for example Arbarello et al. [1]. Hence

$$
c_{2}(\text { End } \mathbf{E})=-\left((2-2 g-n) \sigma+(2 g+n-1-2 d) \sigma+\eta+\sum_{j=1}^{2 g} \xi_{j} e_{j}-u\right)^{2} .
$$

Using the identity $\left(\sum \xi_{j} e_{j}\right)^{2}=-2 \theta \sigma$ and comparing coefficients with those of (1.5) yields the result.

All our weapons are now prepared, and we are ready to attack the main result. It is not a frontal assault, however. Rather, we begin by computing some relations quite different from the $\rho$-classes.

\section{Some ReCursively DEFInED POLYNOMials In $\alpha, \beta, \gamma$ AND $u$}

The $\Gamma$-invariant subring is at the heart of the larger ring containing it; its structure is the key to that of the whole. Our strategy will therefore be to look first for relations between $\alpha, \beta$, and $\gamma$. The method is curiously roundabout. First, certain complicated polynomials, defined recursively here in $\$ 7$, are shown in $\$ 8$ to be relations, by writing down their equivariant extensions explicitly. Then they are shown to be expressible in terms of the much simpler polynomials $\rho_{r, s, t}^{c}$ by a purely algebraic argument, given in $\$ 9$. Not until 12 does a dimension count show that the $\rho$-classes must all be relations.

One relation which holds automatically in all $\mathcal{M}_{n}$ is $\gamma^{g+1}=0$. This is simply because $\gamma=-2 \sum_{j=1}^{g} \psi_{j} \psi_{j+g}$ and each $\psi_{j}^{2}=0$ by skew-commutativity. It is therefore convenient to view our polynomials as belonging to the ring $R=\mathbb{Q}[\alpha, \beta, \gamma] /\left(\gamma^{g+1}\right)$.

Define polynomials $\xi_{r}^{k}$ by $\xi_{r}^{k}=0$ for $r<0, \xi_{0}^{k}=1$, and

$$
(r+1) \xi_{r+1}^{k}=\alpha \xi_{r}^{k}+(r-2 k) \beta \xi_{r-1}^{k}+2 \gamma \xi_{r-2}^{k}
$$

for $r>0$. When $k=0$, these are the polynomials $\xi_{r}$ defined by Zagier [33], and his generating function for the $\xi_{r}$ extends readily.

$$
\begin{aligned}
& \text { Define } F_{0}^{k}(x)=\sum_{r=0}^{\infty} \xi_{r}^{k} x^{r} \in R[[x]] \text {. Then } \\
& F_{0}^{k}(x)=\left(1-\beta x^{2}\right)^{(2 k-1) / 2} e^{-2 \gamma x / \beta}\left(\frac{1+x \sqrt{\beta}}{1-x \sqrt{\beta}}\right)^{(\alpha \beta+2 \gamma) / 2 \beta \sqrt{\beta}} .
\end{aligned}
$$

Proof. From Proposition 4 of Zagier [33] we know that $F_{0}^{0}$ satisfies the differential equation

$$
\left(1-\beta x^{2}\right)\left(F_{0}^{0}\right)^{\prime}(x)=\left(\alpha+\beta x+2 \gamma x^{2}\right) F_{0}^{0}(x) .
$$

Now (7.1) is equivalent to

$$
(r+1) \xi_{r+1}^{k}-(r-1) \beta \xi_{r-1}^{k}=\alpha \xi_{r}^{k}+(1-2 k) \beta \xi_{r-1}+2 \gamma \xi_{r-2},
$$

which shows that $F_{0}^{k}$ satisfies the differential equation

$$
\left(1-\beta x^{2}\right)\left(F_{0}^{k}\right)^{\prime}(x)=\left(\alpha+(1-2 k) \beta x+2 \gamma x^{2}\right) F_{0}^{k}(x),
$$


with initial condition $F_{0}^{k}(0)=1$. But $\left(1-\beta x^{2}\right)^{k} F_{0}^{0}$ satisfies the same differential equation

$$
\begin{aligned}
(1- & \left.\beta x^{2}\right)\left(\left(1-\beta x^{2}\right)^{k} F_{0}^{0}\right)^{\prime} \\
& =\left(1-\beta x^{2}\right)^{k}\left(1-\beta x^{2}\right)\left(F_{0}^{0}\right)^{\prime}-k \beta x\left(1-\beta x^{2}\right)^{k} F_{0}^{0} \\
& =\left(\alpha+\beta x+2 \gamma x^{2}\right)\left(1-\beta x^{2}\right)^{k} F_{0}^{0}-2 k \beta x\left(1-\beta x^{2}\right)^{k} F_{0}^{0} \\
& =\left(\alpha+(1-2 k) \beta x+2 \gamma x^{2}\right)\left(1-\beta x^{2}\right)^{k} F_{0}^{0},
\end{aligned}
$$

and certainly $\left(1-\beta 0^{2}\right)^{k} F_{0}^{0}(0)=1$, so we conclude that

$$
F_{0}^{k}(x)=\left(1-\beta x^{2}\right)^{k} F_{0}^{0}(x) .
$$

Now substitute Zagier's generating function for $F_{0}^{0}$.

$$
\text { The polynomial } \xi_{r}^{k} \text { is a relation on } \mathcal{N} \text { whenever } r \geq g+2 k \text {. }
$$

Proof. An equivalent form of (7.2) is $\xi_{r}^{k}=\sum_{i=0}^{k} \xi_{r-2 i}^{0}(-\beta)^{i}$, and Zagier shows that $\xi_{r}^{0}$ is a relation on $\mathcal{N}$ for $r \geq g$.

Now define an expression with one more index:

$$
\xi_{r, s}^{k}=\sum_{i=0}^{s}\left(\begin{array}{c}
r-2 k+s-i \\
r-2 k
\end{array}\right) \beta^{s-i} \frac{(2 \gamma)^{i}}{i !} \xi_{r-i}^{k} .
$$

Note that this is 0 if $r<2 k$. Moreover, the $i$ th term in the sum vanishes when $i>r$ and also (as an element of $R$ ) when $i>g$. Hence in particular $\xi_{2 k, g+l}^{k}=\beta^{l} \xi_{2 k, g}^{k}$ in $R$ for all $l \geq 0$.

$$
\text { Let } F^{k}(x, y)=\sum_{r, s=0}^{\infty} \xi_{r, s}^{k} x^{r} y^{s} \in R[[x, y]] \text {. Then }
$$

$$
F^{k}(x, y)=\left((1-\beta y)^{2}-\beta x^{2}\right)^{(2 k-1) / 2} e^{-2 \gamma x / \beta}\left(\frac{1+x \sqrt{\beta}-\beta y}{1-x \sqrt{\beta}-\beta y}\right)^{(\alpha \beta+2 \gamma) / 2 \beta \sqrt{\beta}} .
$$

Proof. For fixed $r$ we have

$$
\begin{aligned}
\sum_{s \geq 0} \xi_{r, s}^{k} y^{s} & =\sum_{i=0}^{r} \frac{(2 \gamma y)^{i}}{i !} \xi_{r-i}^{k} \sum_{s=i}^{\infty}\left(\begin{array}{c}
r-2 k+s-i \\
r-2 k
\end{array}\right)(\beta y)^{s-i} \\
& =\frac{1}{(1-\beta y)^{r-2 k+1}} \sum_{i=0}^{r} \frac{(2 \gamma y)^{i}}{i !} \xi_{r-i}^{k} .
\end{aligned}
$$

Multiplying by $x^{r}$ and summing over $r \geq 0$ we obtain

$$
F^{k}(x, y)=(1-\beta y)^{2 k-1} e^{2 \gamma x y /(1-\beta y)} F_{0}^{k}\left(\frac{x}{1-\beta y}\right),
$$

and the desired result follows by substituting the formula given in (7.2).

$$
\begin{aligned}
& \text { For } r, s \geq 0 \text { we have } \\
& \qquad \xi_{r, s}^{k}=\sum_{l=0}^{s}(-1)^{s-l}\left[\left(\begin{array}{c}
r+l \\
r
\end{array}\right)+\left(\begin{array}{c}
r+l-1 \\
r
\end{array}\right)\right] \xi_{s-l}^{k} \xi_{r+s+l}^{k},
\end{aligned}
$$

where the binomial coefficient $\left(\begin{array}{c}-1 \\ 0\end{array}\right)$ is to be taken as 0 .

Proof. Similar to Zagier's Proof 1 of his Theorem 4. 


\section{PRoOf That THE RECURSIVELY DEFINED POLYNOMIALS ARE RELATIONS}

After this algebraic preparation, we now find some relations between $\alpha, \beta$, and $\gamma$ that can be expressed in terms of the classes $\xi_{r, s}^{k}$ introduced above. We make fundamental use of Kirwan's theorem (3.1)(iii), which tells us that any polynomial in the generators that vanishes on the equivariant cohomology of each component of the fixed-point set must be a relation.

(8.1) For $n \geq 0$, let $p \in R[u]$ be an equivariant relation on $\mathcal{M}_{n+2}$, that is, an element of the kernel of the natural map to $H_{T}^{*}\left(\mathcal{M}_{n+2}\right)$. Then $\partial p / \partial u$ is an equivariant relation on $\mathcal{M}_{n}$.

Proof. By Kirwan's theorem (3.1)(iii), it suffices to show that $\partial p / \partial u$ restricts to an equivariant relation on each component of the fixed-point set of $\mathcal{M}_{n}$.

For $F_{0}=\mathcal{N}$, this is obvious, since $p$ is also a relation on $H_{T}^{*}(\mathcal{N})=H^{*}(\mathcal{N})[u]$.

As for $F_{d}$ with $d>0$, we may work in $H^{*}\left(C_{m}\right)$, where $m=2 g+n-1-2 d$. The relation $p$ restricts to a relation in $H^{*}\left(C_{m+2}\right)[u]$; moreover, since by (6.1) the restrictions of $\alpha, \beta, \gamma$ are polynomials in $\eta-u$ and $\theta$, the relation can be expressed as a polynomial $\left.r(\phi, \theta, u)\right|_{\phi=\eta-u}$ such that $\partial r /\left.\partial u(\phi, \theta, u)\right|_{\phi=\eta-u}$ is the restriction of $\partial p / \partial u$, which we want to vanish.

The assignment $u \mapsto \eta-\phi$ induces an isomorphism $H^{*}\left(C_{m+2}\right)[u] \cong H^{*}\left(C_{m+2}\right)[\phi]$, so $r(\phi, \theta, \eta-\phi)$ is a relation in $H^{*}\left(C_{m+2}\right)[\phi]$. Observe now that the derivative with respect to $\eta$ of any relation in $H^{*}\left(C_{m+2}\right)$ is a relation in $H^{*}\left(C_{m}\right)$. This follows directly from the list of relations given by Macdonald [20, 6.21]. Therefore $\partial / \partial \eta(r(\phi, \theta, \eta-\phi))$ is a relation in $H^{*}\left(C_{m}\right)[\phi]$, and hence

$$
\left.\frac{\partial}{\partial \eta}(r(\phi, \theta, \eta-\phi))\right|_{\phi=\eta-u}=\left.\frac{\partial r}{\partial u}(\phi, \theta, u)\right|_{\phi=\eta-u}
$$

is a relation in $H^{*}\left(C_{m}\right)$, as desired.

(8.2) For $n \geq 0$, let $p \in R[u]$ be an equivariant relation on $\mathcal{M}_{n}$. Then $\left(u^{2}-\beta\right) p$ is an equivariant relation on $\mathcal{M}_{n+1}$.

Proof. Let $F_{d}$ be any component of the fixed-point set of $\mathcal{M}_{n+1}$. We show that restricting $\left(u^{2}-\beta\right) p$ to $F_{d}$ yields 0 . It is clearly true on $F_{0}=\mathcal{N}$ since this is contained in $\mathcal{M}_{n}$. For $d>0$, the restriction of $u^{2}-\beta$ to $F_{d}$ is $\eta(2 u-\eta)$ by (6.1). On the other hand $p$ restricted to $F_{d} \cap \mathcal{M}_{n}$ is supposed to be zero. But the image of the inclusion $F_{d} \cap \mathcal{M}_{n} \subset F_{d}$ is Poincaré dual to $\eta$, since it is an étale cover of the inclusion $C_{2 g+n-1-2 d} \subset C_{2 g-2 d+n}$. Hence $\eta$ times a relation on $F_{d} \cap \mathcal{M}_{n}$ is a relation on $F_{d}$.

These results suggest that, even if we are interested only in the relations on $\mathcal{M}_{0}$, it is useful to study $\mathcal{M}_{n}$ for all $n$.

$$
\begin{gathered}
\text { For } n \geq 0 \text { and } k=0, \ldots,[n / 2] \text {, the equivariant class } \\
\qquad F^{k}(u, 1)_{2 g+2 n}=\sum_{r=0}^{g+n} \xi_{r, g+n-r}^{k} u^{r}
\end{gathered}
$$

is an equivariant relation on $\mathcal{M}_{n+2}$.

Proof. By Kirwan's theorem (3.1) (iii), it suffices to show that it restricts to a relation on each component of the fixed-point set of $\mathcal{M}_{n+2}$. 
For the first component $F_{0}$, namely $\mathcal{N}$, this follows immediately from (7.6) and (77.3).

For the remaining components $F_{d}$ with $d>0$, use (6.1) to restrict (7.5) to $F_{d}$. This yields

$$
F^{k}(u, 1)_{2 g+2 n}=\left(e^{\theta u} \frac{(1-(\eta-u)(\eta-2 u))^{d+k-1}}{(1-\eta(\eta-u))^{d-k}}\right)_{2 g+2 n},
$$

where the subscript, as in the past, denotes the part in total degree $2 g+2 n$, that is, in ordinary degree $2(2 g+2 n)$. To show that this vanishes in $H^{*}\left(C_{2 g-2 d+1+n}\right) \subset$ $H^{*}\left(F_{d}\right)$, express it as

$$
\begin{aligned}
& \left(e^{\theta u} \frac{(1-(\eta-u)(\eta-2 u))^{d+k-1}}{(1+\eta u)^{d-k}\left(1-\frac{\eta^{2}}{1+\eta u}\right)^{d-k}}\right)_{2 g+2 n} \\
& =\left(\sum_{i=1}^{\infty}\left(\begin{array}{c}
d-k+i \\
i
\end{array}\right) \frac{\eta^{2 i} e^{\theta u}}{(1+\eta u)^{d-k+i}}(1-(\eta-u)(\eta-2 u))^{d+k-1}\right)_{2 g+2 n}
\end{aligned}
$$

It follows immediately from (5.1) that

$$
\left(\frac{e^{\theta u}(\eta u)^{2 i}}{(1+\eta u)^{d-k+i}}\right)_{2(g-d+n+i+1-k)+j}=0
$$

for $j \geq 0$ (the 2 appearing in the subscript since $\eta u$ and $\theta u$ are substituted for $\eta$ and $\theta$ ) and hence that

$$
\left(\frac{e^{\theta u} \eta^{2 i}}{(1+\eta u)^{d-k+i}}\right)_{2(g-d+n+1-k)+j}=0
$$

for $n \geq 0$. Consequently each term in the sum above vanishes in total degree $2 g+2 n$.

(a) For even $n \geq 0$ and $k=0, \ldots, n / 2$, the equivariant class

$$
\left(\left(2+u^{2}-\beta\right)^{n / 2-k} F^{k}(u, 1)\right)_{2 g+n+2 k}
$$

is an equivariant relation on $\mathcal{M}_{n+2}$ divisible by $u^{2 k}$.

(b) For odd $n \geq 0$ and $k=0, \ldots,(n-1) / 2$, the equivariant class

$$
\left(\left(1+u^{2}-\beta\right)\left(2+u^{2}-\beta\right)^{(n-1) / 2-k} F^{k}(u, 1)\right)_{2 g+n+2 k+1}
$$

is an equivariant relation on $\mathcal{M}_{n+2}$ divisible by $u^{2 k+1}$. 
Proof. Since $u$ is not a zero-divisor in $H_{T}^{*}\left(\mathcal{M}_{n+2}\right)$, to show the expression in (a) is a relation, it suffices to do the same for the part in total degree $2 g+2 n$ of

$$
\begin{aligned}
u^{n-2 k} & \left(2+u^{2}-\beta\right)^{n / 2-k} F^{k}(u, 1) \\
& =\left(\left(1+u^{2}-\beta\right)^{2}-\left((1-\beta)^{2}-\beta u^{2}\right)\right)^{n / 2-k} F^{k}(u, 1) \\
& =\sum_{i}\left(\begin{array}{c}
\frac{n}{2}-k \\
i
\end{array}\right)\left(1+u^{2}-\beta\right)^{2 i}\left((1-\beta)^{2}-\beta u^{2}\right)^{n / 2-k-i} F^{k}(u, 1) \\
& =\sum_{i}\left(\begin{array}{c}
\frac{n}{2}-k \\
i
\end{array}\right)\left(1+u^{2}-\beta\right)^{2 i} F^{n / 2-i}(u, 1) \\
& =\sum_{i, j}\left(\begin{array}{c}
\frac{n}{2}-k \\
i
\end{array}\right)\left(\begin{array}{c}
2 i \\
j
\end{array}\right)\left(u^{2}-\beta\right)^{j} F^{n / 2-i}(u, 1) .
\end{aligned}
$$

By (8.3), $F^{n / 2-i}(u, 1)_{2 g+2 n-2 j}$ is a relation on $\mathcal{M}_{n-j+2}$; hence by (8.2),

$$
\left(\left(u^{2}-\beta\right)^{j} F^{n / 2-i}(u, 1)\right)_{2 g+2 n}
$$

is a relation on $\mathcal{M}_{n+2}$.

The statement about divisibility is easy, since $F^{k}(u, 1)=\sum \xi_{r, s}^{k} u^{r}$ and $\xi_{r, s}^{k}=0$ for $r<2 k$.

The proof of (b) is similar: first multiply by $u^{n-1-2 k}$, compute as before

$$
\begin{array}{r}
u^{n-1-2 k}\left(1+u^{2}-\beta\right)\left(2+u^{2}-\beta\right)^{(n-1) / 2-k} F^{k}(u, 1) \\
=\sum_{i, j}\left(\begin{array}{c}
\frac{n-1}{2}-k \\
i
\end{array}\right)\left(\begin{array}{c}
2 i+1 \\
j
\end{array}\right)\left(u^{2}-\beta\right)^{j} F^{(n-1) / 2-i}(u, 1),
\end{array}
$$

and then apply (8.2) and (8.3).

As in (a), this is clearly divisible by $u^{2 k}$, but the quotient is further divisible by another factor of $u$. This is because the coefficient of $u^{0}$ in the quotient is

$$
\begin{aligned}
& \left((1-\beta)(2-\beta)^{(n-1) / 2-k} \sum_{s=0}^{\infty} \xi_{2 k, s}^{k}\right)_{2 g+n+1} \\
& =\left((1-\beta)(2-\beta)^{(n-1) / 2-k} \sum_{s=g}^{\infty} \xi_{2 k, s}^{k}\right)_{2 g+n+1} \\
& =\left((1-\beta)(2-\beta)^{(n-1) / 2-k} \xi_{2 k, g}^{k} \sum_{l=0}^{\infty} \beta^{l}\right)_{2 g+n+1} \\
& =\left((2-\beta)^{(n-1) / 2-k} \xi_{2 k, g}^{k}\right)_{2 g+n+1} \\
& =0
\end{aligned}
$$

using $\xi_{2 k, g+l}^{k}=\beta^{l} \xi_{2 k, g}^{k}$.

(8.5) For $n \geq-2$ even (resp. odd), $\xi_{r, s}^{k}$ (resp. $\xi_{r, s}^{k}-\beta \xi_{r, s-1}^{k}$ ) is a relation in the ordinary cohomology of $\mathcal{M}_{n+2}$

(i) for $k=[n / 2]-i, r=n-2 i$, and $s=g+i$, where $i=0, \ldots,[n / 2]$;

(ii) for $k=[n / 2]+j, r=n+3 j$, and $s=g-j$, where $j=1, \ldots, g$.

Proof. Suppose first that $n$ is even. For (i), just take the formula from (8.4)(a) with $k=n / 2-i$, plug in $F^{k}(u, 1)=\sum \xi_{r, s}^{k} u^{r}$, divide by $u^{2 k}$ and set $u=0$. Then 
compute using the definition of $\xi_{r, s}^{k}$, the binomial theorem, and $\gamma^{g+1}=0$. For (ii), take the same formula on $\mathcal{M}_{n+2 j}$ with $k=n / 2+j$, apply (8.1) $j$ times, and proceed as in (i). Now suppose that $n$ is odd. It suffices to prove the same statement for the class $\xi_{r, s}^{k}-\beta \xi_{r, s-1}^{k}+\xi_{r-2, g+1}^{k}$, because in case (i) the last term vanishes altogether, and in case (ii) it was shown in the even case to be a relation on $\mathcal{M}_{n+3} \supset \mathcal{M}_{n+2}$. Then everything is similar to the even case.

\section{EXPRESSING THE $\xi$-CLASSES IN TERMS OF THE $\rho$-CLASSES}

We now have many relations on $\mathcal{M}_{n}$. We cannot yet show that the simple polynomials $\rho_{r, s, t}^{c}$ of the main theorem are relations, but at least we can show that the relations we do have are linear combinations of them. Hence the goal of this section is to prove the following purely algebraic result.

(a) For $r \geq 2 k, \xi_{r, s}^{k}$ is a linear combination of those $\rho_{u, v, w}^{r-2 k+v-w}$ with $w \leq r-2 k$ and $u+3 w \leq r$. (b) For $r \geq 2 k+1, \xi_{r, s}^{k}-\beta \xi_{r, s-1}^{k}$ is a linear combination of those $\rho_{u, v, w}^{r-2 k+1+v-w}$ with $w \leq r-2 k+1$ and $u+3 w \leq r$.

It is an easy matter to check, using high-school algebra and the equality of total degrees $r+2 s+3 t=u+2 v+3 w$, that when $n, k, r$ and $s$ are as in (8.5), the conditions (2.4) of membership in $I_{n}^{g}$ are satisfied by the $\rho$-classes named above. Hence the relations of 8.5 belong to $I_{n}^{g}$.

The proof of (9.1) will use a generating function for the $\xi_{r}^{k}$ which generalizes a formula for the $\xi_{r}$ stated without proof in Zagier's paper 33. Zagier kindly communicated a proof to us, and it goes through almost verbatim for the generalization.

$$
\phi_{m}^{k}(r, p)=\operatorname{Coeff}_{x^{m}} \frac{1}{\cosh ^{2 k} \sqrt{3 x}} \frac{\sqrt{3 x}}{\sinh \sqrt{3 x}}\left(\frac{\sqrt{3 x}}{\tanh \sqrt{3 x}}\right)^{r}\left(\frac{1}{x}-\frac{\tanh \sqrt{3 x}}{x \sqrt{3 x}}\right)^{p},
$$

then for $r \geq 0$,

$$
\xi_{r}^{k}=\sum_{m, p} \frac{\phi_{m}^{k}(r, p)}{3^{m+p}(r-2 m-3 p) ! p !} \alpha^{r-2 m-3 p} \beta^{m}(2 \gamma)^{p} .
$$

Proof. The formula for $\phi_{m}^{k}(r, p)$ may be abbreviated as $\operatorname{Coeff}_{x^{m}} A(x) B(x)^{r} C(x)^{p}$. This directly gives a generating function for these numbers with $r$ and $p$ fixed, but to compute

$$
F_{0}^{k}(t)=\sum_{r} \xi_{r}^{k} t^{r}
$$

we need instead a generating function for $\phi_{m}^{k}(l+2 m+3 p, p)$ with $l$ and $p$ fixed and $m$ variable. The passage from one to the other, as usual, is by residue calculus: write $\phi_{m}^{k}(r, p)$ as $\operatorname{Res}_{x=0}\left(A(x) B(x)^{r} C(x)^{p} d x / x^{m+1}\right)$ and change variables to $y=$ $x / B(x)^{2}=(1 / 3) \tanh ^{2}(\sqrt{3 x})$ to get

$$
\phi_{m}^{k}(l+2 m+3 p, p)=\operatorname{Res}_{y=0}\left(a(y) b(y)^{l} c(y)^{p} d y / y^{m+1}\right)
$$

with $a(y)=A(x(y)) x^{\prime}(y) / B(y)^{2}, b(y)=B(x(y)), c(y)=C(x(y)) B(x(y))^{3}$. In other words,

$$
\sum_{m} \phi_{m}^{k}(l+2 m+3 p, p) y^{m}=a(y) b(y)^{l} c(y)^{p}
$$


Then we need to verify

$$
\begin{aligned}
F_{0}^{k}(t) & =\sum_{l, m, p \geq 0} \phi_{m}^{k}(l+2 m+3 p, p) \frac{(\alpha t)^{l}}{l !}\left(\beta t^{2} / 3\right)^{m} \frac{\left(2 \gamma t^{3} / 3\right)^{p}}{p !} \\
& =\sum_{l, p \geq 0} a(y) \frac{(\alpha t b(y))^{l}}{l !} \frac{\left(\gamma t^{3} c(y) / 3\right)^{p}}{p !} \\
& =a(y) \exp \left(\alpha t b(y)+\gamma t^{3} c(y) / 3\right)
\end{aligned}
$$

with $y=\beta t^{2} / 3$. Substituting for $a, b$, and $c$ the formulas above, we find

$$
F_{0}^{k}(t)=\cosh ^{1-2 k}(\sqrt{3 x}) \exp \left((\alpha \beta+2 \gamma) \sqrt{3 x / \beta^{3}}-2 \gamma \tanh (\sqrt{3 x}) / \beta^{3 / 2}\right)
$$

which, since the new variable $t$ is related to the original variable $x$ by $t=\sqrt{3 y / \beta}=$ $\beta^{-1 / 2} \tanh (\sqrt{3 x})$, is equivalent to (7.2).

Proof of (9.1). Consider first part (a). Regarded as a polynomial in $\alpha$ and $\gamma$ only, each $\rho_{u, v, w}^{r-2 k+v-w}$ is homogeneous of degree $u+w$. So let us decompose $\xi_{r, s}^{k}$ likewise into its homogeneous summands relative to this $\alpha, \gamma$-grading. They are nonzero only in $\alpha, \gamma$-degree $r-2 m$ for $m \geq 0$. Indeed, using (9.2) and (7.4), we find that the part of $\xi_{r, s}^{k}$ in $\alpha, \gamma$-degree $r-2 m$ equals

$$
\frac{1}{3^{m}} \sum_{i, j} \frac{(r-2 k+s-i) !}{(r-2 k) !} \frac{\alpha^{r-2 m-j}}{(r-2 m-j) !} \frac{\beta^{s+m-j}}{(s-i) !} \frac{(2 \gamma)^{j}}{i !(j-i) !} \phi_{m-j+i}^{k}(r-i, j-i),
$$

where we adopt the convention of summing over those indices where the factorials all have nonnegative arguments.

The $\rho$-classes having total degree $u+2 v+3 w=r+2 s$ and $\alpha, \gamma$-degree $u+w=$ $r-2 m$ are of the form $\rho_{r-2 m-w, s^{\prime}-w, w}^{r^{\prime}+s^{\prime}-2 w}$ for $w=0, \ldots, \min \left(\left[\left(r^{\prime}+s^{\prime}\right) / 2\right], s^{\prime}, r-2 m\right)$, where we have introduced the abbreviations $r^{\prime}=r-2 k$ and $s^{\prime}=s+m$. Using their definition (2.3), we may express any linear combination of these $\rho$-classes as

$$
\begin{aligned}
& \frac{1}{3^{m}} \sum_{i, w} a_{w}(q-2 w-i) ! \frac{\alpha^{r-i-2 m-w}}{(r-i-2 m-w) !} \frac{\beta^{s^{\prime}-i-w}}{\left(s^{\prime}-i-w\right) !} \frac{(2 \gamma)^{i+w}}{i !} \\
= & \frac{1}{3^{m}} \sum_{j, w} a_{w}(q-w-j) ! \frac{\alpha^{r-2 m-j}}{(r-2 m-j) !} \frac{\beta^{s^{\prime}-j}}{\left(s^{\prime}-j\right) !} \frac{(2 \gamma)^{j}}{(j-w) !},
\end{aligned}
$$

where $a_{w}$ are arbitrary scalars, $q=r^{\prime}+s^{\prime}$, and the factor of $1 / 3^{m}$ is inserted for convenience.

At least when $s$ is large enough that $s^{\prime}$ and $\left[\left(r^{\prime}+s^{\prime}\right) / 2\right] \geq r-2 m$, these span all the polynomials in $\alpha, \beta, \gamma$ of the given total degree and $\alpha, \gamma$-degree. It is therefore possible to write the part of $\xi_{r, s}^{k}$ in $\alpha, \gamma$-degree $r-2 m$ as a linear combination of this kind. The goal is to show that $a_{w}=0$ whenever either $w>r^{\prime}$ or $u+3 w>r$, that is, $w>m$.

The reader may worry that this will only prove the desired result for $s$ large compared to $r$. But, according to [2.3) and [7.4), the coefficient, in all of the polynomials we are concerned with, of the monomial $\alpha^{a} \beta^{s-b} \gamma^{c}$ for fixed $a, b, c$ is a rational function of $s$. So if a linear dependence between these polynomials can be established for sufficiently large $s$, then it holds for all $s$. 
To determine the scalars $a_{w}$, set the coefficients of $\alpha^{r-2 m-j} \beta^{s^{\prime}-j}(2 \gamma)^{j}$ in the last two equations to be equal:

$$
\sum_{i} \frac{\left(r^{\prime}+s-i\right) !\left(s^{\prime}-j\right) !}{r^{\prime} !(s-i) ! i !(j-i) !} \phi_{m-j+i}^{k}(r-i, j-i)=\sum_{w} \frac{a_{w}(q-w-j) !}{(j-w) !} .
$$

Let $b_{j}$ be the left-hand side, and let $L$ be the lower triangular matrix whose $(j, w)$ entry is $(q-w-j) ! /(j-w)$ !. Here $j, w$ index the rows and columns and run from 0 to $r-2 m$. In vector notation, the equation above then says $\left(b_{j}\right)=L\left(a_{w}\right)$.

The inverse of $L$ is the lower triangular matrix whose $(w, j)$ entry is

$$
(-1)^{w+j} \frac{(q+1-2 w)}{(w-j) !(q+1-w-j) !} .
$$

Indeed, the sum that needs to be demonstrated is

$$
\sum_{j=w}^{w^{\prime}}(-1)^{w^{\prime}+j} \frac{\left(q+1-2 w^{\prime}\right)(q-w-j) !}{\left(w^{\prime}-j\right) !\left(q+1-w^{\prime}-j\right) !(j-w) !}=\delta_{w, w^{\prime}}
$$

This is obvious for $w \geq w^{\prime}$. For $w<w^{\prime}$, if the summand is denoted $N_{j}$, then as Shalosh B. Ekhad has pointed out [7],

$\left(q+1-w-w^{\prime}\right)\left(w^{\prime}-w\right) N_{j}=(j-w)(q+1-w-j) N_{j}-(j+1-w)(q-w-j) N_{j+1} ;$

since the coefficient on the left is independent of $j$ and is nonzero for large $s$ (and hence for large $q$ ), the sum telescopes.

Hence

$$
a_{w}=\sum_{i, j} \frac{(-1)^{w-j}(q+1-2 w)\left(r^{\prime}+s-i\right) !\left(s^{\prime}-j\right) !}{(w-j) !(q+1-w-j) ! r^{\prime} !(s-i) ! i !(j-i) !} \phi_{m-j+i}^{k}(r-i, j-i) .
$$

Now sum over all variables, and group the factorials together as binomial coefficients, to create the grand generating function

$$
\begin{aligned}
& \sum_{m, q, s, w} a_{w} \frac{(q+1-w) !}{(q+1-2 w)\left(s^{\prime}-w\right) !} M^{m} Q^{q} S^{s} W^{w} \\
& =\sum_{i, j, m, q, s, w}(-1)^{w-j}\left(\begin{array}{c}
r^{\prime}+s-i \\
r^{\prime}
\end{array}\right)\left(\begin{array}{c}
s^{\prime}-j \\
w-j
\end{array}\right)\left(\begin{array}{c}
+1-w \\
j
\end{array}\right)\left(\begin{array}{l}
j \\
i
\end{array}\right) \phi_{m-j+i}^{k}(r-i, j-i) M^{m} Q^{q} S^{s} W^{w} .
\end{aligned}
$$

Using the binomial series, we can successively eliminate the sums over $q$, $w$, and $s$, to obtain

$$
\sum_{i, p, m}\left(\begin{array}{c}
i+p \\
i
\end{array}\right) \frac{(1-W Q)^{m}\left(W Q^{2}\right)^{i+p} M^{p+m} S^{i}}{Q(1-Q)^{i+p+1}(1-S(1-W Q))^{r^{\prime}+1}} \phi_{m}^{k}(r-i, p) .
$$

Substituting (9.2) for the sum over $m$ yields

$$
\begin{aligned}
& \frac{1}{Q(1-S(1-W Q))^{r^{\prime}+1}} \sum_{i, p}\left(\begin{array}{c}
i+p \\
i
\end{array}\right) \frac{\left(W Q^{2}\right)^{i+p} M^{p} S^{i}}{(1-Q)^{i+p+1}} \\
& \cdot \frac{1}{\cosh ^{2 k} \sqrt{X}} \frac{\sqrt{X}}{\sinh \sqrt{X}}\left(\frac{\sqrt{X}}{\tanh \sqrt{X}}\right)^{r-i}\left(\frac{3}{X}-\frac{3 \tanh \sqrt{X}}{X \sqrt{X}}\right)^{p},
\end{aligned}
$$


where $X=3(1-W Q) M$. Applying the binomial theorem again and simplifying transforms this to

$$
\begin{aligned}
& \frac{1}{Q(1-S(1-W Q))^{r^{\prime}+1}} \frac{1-W Q}{1-Q-W Q+W Q^{2}(1-S(1-W Q)) \frac{\tanh \sqrt{X}}{\sqrt{X}}} \\
& \cdot \frac{1}{\cosh ^{2 k} \sqrt{X}} \frac{\sqrt{X}}{\sinh \sqrt{X}}\left(\frac{\sqrt{X}}{\tanh \sqrt{X}}\right)^{r} .
\end{aligned}
$$

The goal is to show that the coefficient of $M^{m} Q^{q} S^{s} W^{w}$ vanishes in the above for $q=r^{\prime}+s^{\prime}$ and $w>\min \left(r^{\prime}, m\right)$. Since $X=3(1-W Q) M$, it is equivalent to multiply the generating function $(9.3)$ by $3^{m}(1-W Q)^{m}$ and take the coefficient of $X^{m} Q^{q} S^{s} W^{w}$. But the second line of (9.3) is a power series in $X$ only, so this coefficient is a linear combination of the coefficients, for $n \leq m$, of $X^{n} Q^{q} S^{s} W^{w}$ in

$$
\frac{1}{Q(1-S(1-W Q))^{r^{\prime}+1}} \frac{(1-W Q)^{m+1}}{1-Q-W Q+W Q^{2}(1-S(1-W Q)) \frac{\tanh \sqrt{X}}{\sqrt{X}}} .
$$

We will show that these all vanish.

In fact, we may replace $\tanh \sqrt{X} / \sqrt{X}$ by $1+X$ in the above, for this can be undone by substituting a power series of the form $c_{1} X+c_{2} X^{2}+\cdots$ for $X$. Hence the coefficients of $X^{n} Q^{q} S^{s} W^{w}$ in the former are linear combinations of $X^{p} Q^{q} S^{s} W^{w}$ in the latter, for $p \leq m$.

Taking coefficients of $X^{p}, S^{s}, W^{w}$, and $Q^{r^{\prime}+s^{\prime}}$ in the resulting rational function yields

$$
\sum_{i=0}^{s}(-1)^{w+i}\left(\begin{array}{c}
r^{\prime}+s-p-i \\
r^{\prime}-p
\end{array}\right)\left(\begin{array}{c}
p+i \\
i
\end{array}\right)\left(\begin{array}{c}
s^{\prime}-p-i \\
w-p-i
\end{array}\right)\left(\begin{array}{c}
r^{\prime}+s^{\prime}+1-w \\
n+i
\end{array}\right)
$$

Let $F(s, i)$ be the $i$ th term in the sum. As pointed out by Shalosh B. Ekhad [7], if we define $G(s, i)$ by

$$
\frac{i\left(r^{\prime}+s+1-p-i\right)\left(s^{\prime}+1-p-i\right)\left(r^{\prime}+s^{\prime}+2-w\right)\left(r^{\prime}+s+s^{\prime}+3-p-w-i\right)}{(s+1-i)\left(s^{\prime}+1-w\right)\left(r^{\prime}+s^{\prime}+2-p-w-i\right)} F(s, i),
$$

then by high-school algebra,

$$
\begin{aligned}
& G(s, i+1)-G(s, i)= \\
& \quad\left(r^{\prime}+s+1-w\right)\left(r^{\prime}+s^{\prime}+2-w\right) F(s, i)-(s+1)\left(r^{\prime}+s^{\prime}+2-p-w\right) F(s+1, i),
\end{aligned}
$$

and so, summing over $i$, we deduce that the sum satisfies a linear recurrence relation in $s$ :

$\left(r^{\prime}+s+1-w\right)\left(r^{\prime}+s^{\prime}+2-w\right) \sum_{i=0}^{s} F(s, i)-(s+1)\left(r^{\prime}+s^{\prime}+2-p-w\right) \sum_{i=0}^{s+1} F(s+1, i)=0$.

If $w>r^{\prime}$ and $p \leq m$, the coefficient of the first sum in the recurrence is 0 for $s=w-r^{\prime}-1$, and the coefficient of the second sum is nonzero for all subsequent $s$. Hence the sum vanishes for all $s$ sufficiently large, namely $\geq w-r^{\prime}$.

If $w>m$ and $p \leq m$, then every term in the sum is easily seen to vanish for $s=0$, and for $s=-r^{\prime}-m-1+p+w$ if this is positive. The recurrence then implies that the sum is 0 for all positive $s$.

This completes the proof of part (a); the proof of (b) is similar. Because

$$
\left(\begin{array}{c}
r^{\prime}+s-i \\
r^{\prime}
\end{array}\right)-\left(\begin{array}{c}
r^{\prime}+s-1-i \\
r^{\prime}
\end{array}\right)=\left(\begin{array}{c}
r^{\prime}-1+s-i \\
r^{\prime}-1
\end{array}\right)
$$


the grand generating function has $r^{\prime}-1$ substituted for $r^{\prime}$; hence the same is true for all subsequent formulas.

\section{The RELATIONS DIVISIBLE BY $\gamma$}

Many $\Gamma$-invariant relations on $\mathcal{M}_{n}$ were computed in 8 , but none of these relations were divisible by $\gamma$. To find some $\Gamma$-invariant relations that are divisible by $\gamma$, we revive the space $\mathcal{M}$ of flat connections, which is diffeomorphic to $\mathcal{M}_{0}$ as described in $\S 2$ of our previous paper [14]. We will find a relationship between the cohomology at genus $g$ and genus $g-1$. Accordingly, we will write $\mathcal{M}^{g}$ to indicate the dependence of $\mathcal{M}$ on the genus. Let $G=\mathrm{SL}(2, \mathbb{C})$, and define $\mu_{g}: G^{2 g} \rightarrow G$ by $\mu_{g}\left(A_{j}, B_{j}\right)=\prod A_{j} B_{j} A_{j}^{-1} B_{j}^{-1}$. Then $\mathcal{M}^{g}=\mu_{g}^{-1}(-I) / G$, where $G$ acts by simultaneous conjugation. The goal of this section is to prove the following.

(10.1) Let $\rho \in \mathbb{Q}\left[\alpha, \beta, \psi_{j}\right]$ be a relation on $\mathcal{M}^{g-1}$. Then $\psi_{j} \psi_{j+g} \rho$ for each $j \leq g$, and hence $\gamma \rho$, are relations on $\mathcal{M}^{g}$.

The proof will involve the following lemma.

(10.2) The only critical value of $\mu_{g}$ is the identity matrix $I$.

Proof. The derivative $d \mu_{g}: \mathfrak{g}^{2 g} \rightarrow \mathfrak{g}$ at $\left(A_{j}, B_{j}\right) \in G^{2 g}$ is easy to compute explicitly: see for example Goldman [8] or Gunning [10, Lemma 26]. It is a sum of $g$ terms, the $k$ th being conjugate to $\left(a_{j}, b_{j}\right) \mapsto\left(I-\operatorname{Ad} A_{k}^{-1}\right) b_{k}-\left(I-\operatorname{Ad} B_{k}^{-1}\right) a_{k}$. At a critical point, then, all $g$ of these maps must fail to surject.

For $A \neq \pm I \in G=\mathrm{SL}(2, \mathbb{C})$, it is easy to check by hand, using Jordan canonical form, that the image of $\left(I-\operatorname{Ad} A^{-1}\right): \mathfrak{g} \rightarrow \mathfrak{g}$ is a 2-dimensional subspace from which the eigenspaces of $A$ can be recovered and that it is a subalgebra if and only if $A$ is not diagonalizable. If the maps are not surjective, then either these 2-dimensional subspaces must coincide, or one of $A_{k}$ or $B_{k}$ is $\pm I$. In the former case, $A_{k}$ and $B_{k}$ must have a common eigenspace (if they are not diagonalizable) or eigenspaces (if they are). In any case, they must commute, and so $\mu_{g}\left(A_{j}, B_{j}\right)=I$.

Notice that the arguments of the last paragraph are special to $\mathrm{SL}(2, \mathbb{C})$; the situation for $\operatorname{SL}(r, \mathbb{C})$ with $r>2$ is more complicated.

Proof of (10.1). Let $K=\mathrm{SU}(2)$, and let $L \subset G$ be the locus of matrices of the form $U^{-1} D U$, where $U \in K$ and $D=\operatorname{diag}(\lambda, 1 / \lambda)$ for some positive real $\lambda$. Then $L$ is a smooth, contractible submanifold of $G$ whose tangent space at the identity is $i$ times that of $K$.

Let $\mathcal{L}$ be the intersection $\mu_{g}^{-1}(-I) \cap\left(G^{2 g-2} \times L^{2}\right)$. This is preserved by the $K$-action, and there are inclusions

$$
\mu_{g-1}^{-1}(-I) \times\{I\} \times\{I\} \subset \mathcal{L} \subset \mu_{g}^{-1}(-I) .
$$

Dividing by the $K$-action yields inclusions

$$
\tilde{\mathcal{M}}^{g-1} \subset \mathcal{L} / K \subset \tilde{\mathcal{M}}^{g}
$$

Here $\tilde{\mathcal{M}}^{g}=\mu_{g}^{-1}(-I) / K$, which is a $G / K$-bundle over $\mathcal{M}^{g}$. Since $G / K$ is contractible, this is homotopy equivalent to $\mathcal{M}^{g}$. It is not hard to check, using the definition of the universal classes in $\S 1$ of our previous paper [14, that $\alpha, \beta$, and $\gamma$, regarded as classes on $\tilde{\mathcal{M}}^{g}$, restrict to their counterparts on $\tilde{\mathcal{M}}^{g-1}$.

It therefore suffices to prove the following two claims: that $\tilde{\mathcal{M}}^{g-1} \subset \mathcal{L} / K$ induces an isomorphism on cohomology and that $\mathcal{L} / K \subset \tilde{\mathcal{M}}^{g}$ is Poincaré dual to $\psi_{g} \psi_{2 g}$. 
For then $\psi_{g} \psi_{2 g} \rho$ must be a relation on $\tilde{\mathcal{M}}^{g}$. The result follows by symmetry, since the action of $\Gamma$ on $\mathbb{Q}\left[\alpha, \beta, \psi_{j}\right]$ certainly preserves the ideal of relations, and there is an element of $\Gamma$ taking $\psi_{g} \psi_{2 g}$ to $\psi_{j} \psi_{j+g}$ for each $j$.

To prove the first claim, first note that $\mathcal{L}$ can be regarded as the fibered product $G^{2 g-2} \times_{G}(L \times L)$, where the map $G^{2 g-2} \rightarrow G$ is $\mu_{g-1}$ and the map $L \times L \rightarrow G$ is $(A, B) \rightarrow-A B A^{-1} B^{-1}$. A direct computation shows that no two $A, B \in L$ have $A B A^{-1} B^{-1}=-I$; hence by (10.2) the map $L \times L \rightarrow G$ never touches a critical value of $\mu_{g-1}$. So $\mathcal{L}$ is locally trivial over $L \times L$ with fiber $\mu_{g-1}^{-1}(-I)$. Since $L \times L$ is contractible, this implies that $\mathcal{L}$ is homeomorphic to $L \times L \times \mu_{g-1}^{-1}(-I)$.

It follows that $\tilde{\mathcal{M}}^{g-1}$ and $\mathcal{L} / K$ are homotopy equivalent. Indeed, they are homotopy equivalent to the homotopy quotients $\left(\mu_{g-1}^{-1}(-I) \times E K\right) / K$ and $(\mathcal{L} \times E K) / K$, respectively, and the latter retracts onto the former, since $B K$ is a direct limit of manifolds and $L \times L$ is contractible.

To prove the second claim, first note that $L$ meets $K \subset G$ transversely at the single point $I$. It is therefore Poincaré dual to the standard generator of $H^{3}(G, \mathbb{Z})$. We can now either imitate the argument given by the second author [31, Prop. 19.3] for the $\mathrm{SU}(2)$ space $\mathcal{N}^{g}=\left(\mu_{g}^{-1}(-I) \cap K^{2 g}\right) / K$ or simply use that result. It tells us that the natural maps in the top row of the diagram

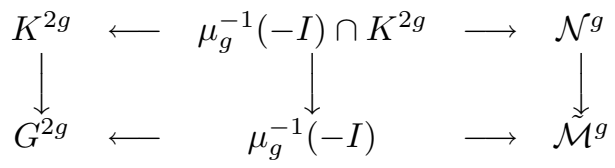

induce isomorphisms on $H^{3}$ under which the generator of the $j$ th copy of $H^{3}(K, \mathbb{Z})$ corresponds to $\psi_{j}$. Since the outer columns also induce isomorphisms on $H^{3}$, so does every map in the diagram.

Since the pull-back by inclusion is Poincaré dual to transverse intersection, it now suffices to check that $G^{2 g-2} \times L^{2}$ is transverse to $\mu_{g}^{-1}(-I)$, or equivalently, that at every point of $\mathcal{L}$ the derivative $d \mu_{g}$ remains surjective when restricted to the tangent space to $G^{2 g-2} \times L^{2}$. But this is true even if we restrict further to the tangent space to $G^{2 g-2}$, since as stated before we are at a regular value of $\mu_{g-1}$.

\section{The Cohomology not FiXed By $\Gamma$}

Everything so far has been about the $\Gamma$-invariant part of $H^{*}\left(\mathcal{M}_{n}\right)^{\Sigma}$, generated by $\alpha, \beta$, and $\gamma$. Now it is time to say something about the noninvariant part and the classes $\psi_{j}$.

We begin with a result relating the noninvariant parts of the cohomology of the symmetric product $C_{m}$ at genus $g$ to the invariant part at lower genera.

(11.1)

As a $\Gamma$-module, the cohomology of the symmetric product $C_{m}^{g}$ has the form

$$
H^{*}\left(C_{m}^{g}\right)=\bigoplus_{k=0}^{g} \Lambda_{0}^{k}(\xi) \otimes \mathbb{Q}[\eta, \theta] / I\left(C_{m-k}^{g-k}\right),
$$

where $I\left(C_{m-k}^{g-k}\right)$ is the ideal of relations between $\eta$ and $\theta$ in $C_{m-k}^{g-k}$, with the convention that $I\left(C_{m-k}^{g-k}\right)=\mathbb{Q}[\eta, \theta]$ if $m-k<0$. 
Proof. As shown in 95 there is a surjection of $\Gamma$-algebras

$$
\bigoplus_{k=0}^{g} \Lambda_{0}^{k}(\xi) \otimes \mathbb{Q}[\eta, \theta] \longrightarrow H^{*}\left(C_{m}^{g}\right)
$$

where $\Lambda_{0}^{k}$, being irreducible, is spanned by the orbit of $\xi_{1} \xi_{2} \cdots \xi_{k}$ under $\Gamma$. It therefore suffices to show that a polynomial $p(\eta, \theta)$ is a relation on $C_{m-k}^{g-k}$ if and only if $p(\eta, \theta) \xi_{1} \xi_{2} \cdots \xi_{k}$ is a relation on $C_{m}^{g}$.

By Poincaré duality the latter is true if and only if for all polynomials $q\left(\eta, \xi_{j}\right)$,

$$
p(\eta, \theta) \xi_{1} \xi_{2} \cdots \xi_{k} q\left(\eta, \xi_{j}\right)\left[C_{m}^{g}\right]=0 .
$$

Now, from the description of $H^{*}\left(C_{m}^{g}\right)$ in Macdonald [20], it follows that $\prod_{j=1}^{2 g} \xi_{j}^{p_{j}} \eta^{q}\left[C_{m}^{g}\right]=1$ if $\sum_{j=1}^{g} p_{j}+q=m$ (so that the degrees match) and $p_{j}=$ $p_{j+g} \leq 1$ for each $j \leq g$ (so that it becomes a monomial in $\eta$ and $\theta_{j}=\xi_{j} \xi_{j+g}$ ). Otherwise it equals 0 . Hence in (11.2) we only need to consider the case

$$
q\left(\eta, \xi_{j}\right)=\xi_{g+1} \cdots \xi_{g+k} r\left(\eta, \theta_{k+1}, \ldots, \theta_{g}\right) .
$$

But $q$ can be averaged with its images under all permutations of $\theta_{k+1}, \ldots, \theta_{g}$ without changing the value of (11.2). Hence we only need to consider the case where $r$ is a polynomial in $\eta$ and $\theta_{k+1}+\cdots+\theta_{g}$. But then

$$
\begin{aligned}
p(\eta, \theta) & \xi_{1} \xi_{2} \cdots \xi_{k} q\left(\eta, \xi_{j}\right)\left[C_{m}^{g}\right] \\
& =(-1)^{k} p(\eta, \theta) \theta_{1} \theta_{2} \cdots \theta_{k} r\left(\eta, \theta_{k+1}+\cdots+\theta_{g}\right)\left[C_{m}^{g}\right] \\
& =(-1)^{k} p\left(\eta, \theta_{k+1}+\cdots+\theta_{g}\right) \theta_{1} \theta_{2} \cdots \theta_{k} r\left(\eta, \theta_{k+1}+\cdots+\theta_{g}\right)\left[C_{m}^{g}\right] .
\end{aligned}
$$

This always vanishes if $k>m$. Otherwise it equals $(-1)^{k} p(\eta, \theta) r(\eta, \theta)\left[C_{m-k}^{g-k}\right]$. This vanishes for all $r$ if and only if $p(\eta, \theta) q\left(\eta, \xi_{j}\right)\left[C_{m-k}^{g-k}\right]$ vanishes for all polynomials $q$ in $\eta$ and $\xi_{j}$, since it does not alter the latter expression to replace $q$ with its projection on the $\Gamma$-invariant part, which is a polynomial in $\eta$ and $\theta$. Again by Poincaré duality, this is equivalent to $p(\eta, \theta)=0$ in $C_{m-k}^{g-k}$.

(11.3) As a $\Gamma$-module, the $\Sigma$-invariant part of the $T$-equivariant cohomology of $\mathcal{M}_{n}^{g}$ has the form

$$
H_{T}^{*}\left(\mathcal{M}_{n}^{g}\right)^{\Sigma} \cong \bigoplus_{k=0}^{g} \Lambda_{0}^{k}(\psi) \otimes \mathbb{Q}[\alpha, \beta, \gamma, u] / I_{T}\left(\mathcal{M}_{n+k}^{g-k}\right) .
$$

Consequently, as a $\Gamma$-module, the $\Sigma$-invariant part of the ordinary cohomology of $\mathcal{M}_{n}^{g}$ has the form

$$
H^{*}\left(\mathcal{M}_{n}^{g}\right)^{\Sigma} \cong \bigoplus_{k=0}^{g} \Lambda_{0}^{k}(\psi) \otimes \mathbb{Q}[\alpha, \beta, \gamma] / I\left(\mathcal{M}_{n+k}^{g-k}\right) .
$$

Proof. First we show that if $\lambda \in \Lambda_{0}^{k}(\psi)$ and $r \in I_{T}\left(\mathcal{M}_{n+k}^{g-k}\right)$, then $\lambda r \in I_{T}\left(\mathcal{M}_{n}^{g}\right)$. Certainly $r$ restricts to relations between $\alpha, \beta, \gamma$ and $u$ on $\mathcal{N}^{g}=F_{0}^{g}$ and between $\eta, \theta$ and $u$ on the remaining fixed components $F_{d}^{g} \subset \mathcal{M}_{n}^{g}$. On the other hand, by Kirwan's theorem (3.1)(iii) it suffices to show that $\lambda r$ restricts to similar relations on the fixed components $F_{d}^{g-k}$ of $\mathcal{M}_{n+k}^{g-k}$.

The case of $d>0$ follows immediately from the lemma. As for $d=0$, Proposition 2.5 of King-Newstead [16] asserts that if $\lambda \in \Lambda_{0}^{k}(\psi)$ and if $r$ is a relation between $\alpha, \beta, \gamma$ on $\mathcal{N}^{g}$, then $\lambda r$ is a relation on $\mathcal{N}^{g-k}$. This is exactly what is needed. 
The left-hand side is therefore a quotient of the right-hand side. To complete the proof, it remains only to check that the $\Sigma$-invariant, $T$-equivariant Poincaré polynomials agree. But if $P=\sum_{i} t^{i} \operatorname{dim} H^{i}$, then

$$
\begin{aligned}
(1 & \left.-t^{2}\right) P_{T}^{\Sigma}\left(\mathcal{M}_{n}^{g}\right) \\
& =P^{\Sigma}\left(\mathcal{M}_{n}^{g}\right) \\
& =P^{\Sigma}\left(\mathcal{N}^{g}\right)+\sum_{d=1}^{g+\left[\frac{n-1}{2}\right]} t^{2 g+2 d-2} P\left(C_{2 g+n-1-2 d}^{g}\right) \\
& =\sum_{k=0}^{g}\left(\left(\begin{array}{c}
2 g \\
k
\end{array}\right)-\left(\begin{array}{c}
2 g \\
k-2
\end{array}\right)\right)\left(t^{3 k} P^{\Sigma}\left(\mathcal{N}^{g-k}\right)+\sum_{d=1}^{g+\left[\frac{n-1}{2}\right]} t^{2 g+2 d-2} t^{k} P\left(C_{2 g+n-1-2 d-k}^{g-k}\right)\right) \\
& =\sum_{k=0}^{g}\left(\left(\begin{array}{c}
2 g \\
k
\end{array}\right)-\left(\begin{array}{c}
2 g \\
k-2
\end{array}\right)\right) t^{3 k}\left(P^{\Sigma}\left(\mathcal{N}^{g-k}\right)+\sum_{d=1}^{g+\left[\frac{n-1}{2}\right]} t^{2(g-k)+2 d-2} P\left(C_{2(g-k)+n-1-2 d+k}^{g-k}\right)\right) \\
& =\sum_{k=0}^{g}\left(\left(\begin{array}{c}
2 g \\
k
\end{array}\right)-\left(\begin{array}{c}
2 g \\
k-2
\end{array}\right)\right) t^{3 k} P^{\Sigma}\left(\mathcal{M}_{n+k}^{g-k}\right) \\
& =\left(1-t^{2}\right) \sum_{k=0}^{g}\left(\left(\begin{array}{c}
2 g \\
k
\end{array}\right)-\left(\begin{array}{c}
2 g \\
k-2
\end{array}\right)\right) t^{3 k} P_{T}^{\Sigma}\left(\mathcal{M}_{n+k}^{g-k}\right),
\end{aligned}
$$

using Kirwan's theorem on the Leray sequence (3.1) (ii) in steps 1 and 6, the perfection of the Morse stratification (3.1) (i) in steps 2 and 5, the lemma and the result of King-Newstead in step 3, and high-school algebra in step 4.

\section{WRAP-UP}

At last we can show that the polynomials $\rho_{r, s, t}^{c}$ of $(2.3)$ are relations, using every tool at our disposal: (9.1), (10.1), 11.3), and a dimension count. Recall that $I_{n}^{g}$ is the ideal of $\rho$-classes introduced in $\S(2)$.

$$
\text { Every element of } I_{n}^{g} \text { is a relation on } \mathcal{M}_{n}^{g} \text {. }
$$

Proof. It is actually more convenient to work with $n+2$ than $n$, so let $n \geq-2$. Then every element of $I_{n+2}^{g}$ has total degree $\geq 2 g+n$. In degree $2 g+n, I_{n+2}^{g}$ is spanned by the elements $\rho_{n-2 i, g+i, 0}^{g+i}$ for $i=0, \ldots,[n / 2]$. The degrees with respect to $\alpha$ are all different, so these are linearly independent. The relations $\xi_{n-2 i, g+i}^{[n / 2]-i}$ of type (i) given in (8.5) are all of degree $2 g+n$, also number $[n / 2]+1$, and are linearly independent for the same reason. By (9.1) they are in the linear span of the $\rho_{n-2 i, g+i, 0}^{g+i}$. Hence this equals the linear span of the relations of type (i), so all the $\rho_{n-2 i, g+i, 0}^{g+i}$ are relations.

The diagram is intended to help the reader visualize the ideal of relations. Each dot represents one of the $\rho_{r, s, t}^{c}$. The total degree is the vertical coordinate, and $r$ is the horizontal coordinate. The two edges of the dotted region reflect the two constraints imposed by (2.4). To avoid having to draw a third dimension, only those relations with $t=0$ have been shown. The dotted region for any fixed $t>0$ would look similar, only translated in a northwesterly direction. The relations established in the previous paragraph are those in the bottom row.

The rest of the proof proceeds by induction on the total degree. We have already seen that the part of $I_{n}^{g}$ in total degree $2 g+n$ consists entirely of relations. Now fix $j>0$ and consider the part of $I_{n+2}^{g}$ in degree $2 g+n+j$. Assume by induction that 


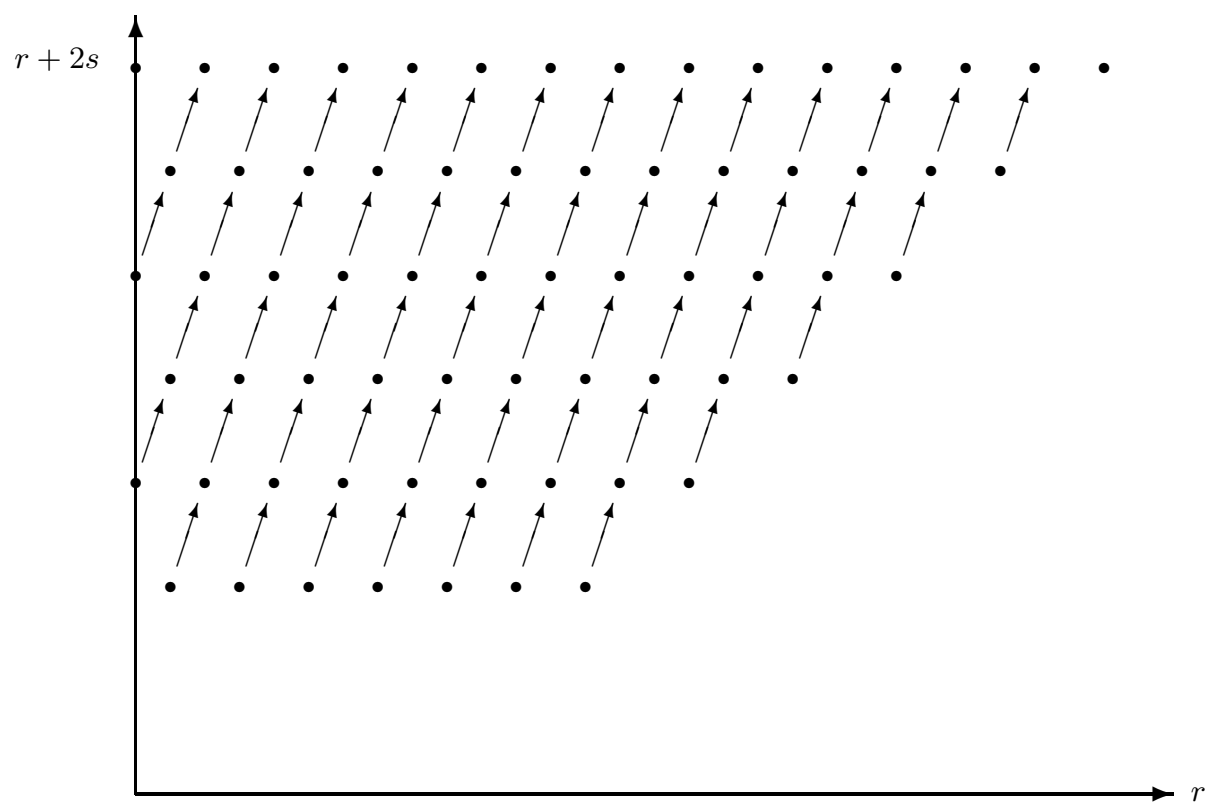

for all $g$ and $n$, the parts of $I_{n+2}^{g}$ in degree $<2 g+n+j$ are known to be relations on $\mathcal{M}_{n+2}^{g}$.

In particular, if $\rho_{r, s, t}^{c} \in I_{n+2}^{g}$ has degree $2 g+n+j$ and $t>0$, then $\rho_{r, s, 0}^{c} \in$ $I_{n+2}^{g-t}$ has degree $2(g-t)+n+(j-t)$ and hence is a relation on $\mathcal{M}_{n+2}^{g-t}$. By (11.3), $\psi_{1} \cdots \psi_{n+2} \rho_{r, s, 0}^{c}$ is a relation on $\mathcal{M}_{0}^{g-t+n+2}$, so by (10.1),$\psi_{1} \cdots \psi_{n+2} \rho_{r, s, t}^{c}$ is a relation on $\mathcal{M}_{0}^{g+n+2}$, and by (11.3) again, $\rho_{r, s, t}^{c}$ is a relation on $\mathcal{M}_{n+2}^{g}$. Because these relations have $t>0$, they are not shown in the diagram.

Only the $\rho_{r, s, t}^{c}$ with $t=0$ remain. Consider first those relations of the form $\rho_{0, s, 0}^{c}=c ! / s ! \beta^{s} \in I_{n+2}^{g}$. These are the relations at the left-hand edge of the diagram. If the degree equals $2 g+n+j$, then since $j>0$,

$$
2 s=2 g+n+j \geq 2 g-2+(n+3)
$$

and

$$
3 s=3 g+\frac{3}{2} n+\frac{3}{2} j>3 g-3+(n+3),
$$

so in fact $\rho_{0, s, 0}^{c} \in I_{n+3}^{g}$. Since it has degree $2 g+n+j=2 g+(n+1)+(j-1)$, by the induction hypothesis again it is a relation on $\mathcal{M}_{n+3}^{g}$ and hence on $\mathcal{M}_{n+2}^{g} \subset \mathcal{M}_{n+3}^{g}$.

What if $t=0$ but $r>0$ ? It is easily checked that

$$
r \rho_{r, s, 0}^{c}=c \alpha \rho_{r-1, s, 0}^{c-1}+(c-r) \rho_{r-1, s-1,1}^{c-2} .
$$

Now if $r+2 s=2 g+n+j$ and $1 \leq r \leq n+3 j-2$ (so that we are not at the righthand edge of the diagram), we know from the induction hypothesis that $\rho_{r-1, s, 0}^{c-1}$ is a relation. And certainly $\rho_{r, s-1,1}^{c-2}$ is a relation, since it belongs to $I_{n+2}^{g}$ and has $t>0$. Hence under these circumstances $\rho_{r, s, 0}^{c}$ is a relation. This fills in the interior of the diagram; the arrows depict multiplication by $\alpha$ (modulo $\gamma$ ), which takes $\rho_{r-1, s, 0}^{c-1}$ to $\rho_{r, s, 0}^{c}$. 
Only one class of total degree $2 g+n+j$ remains in $I_{n+2}^{g}$. This is $\rho_{n+3 j, g-j}^{g}$, at the right-hand edge of the diagram. We know from (8.5) (ii) that the class $\xi_{n+3 j, g-j}^{[n / 2]+j}$ is a relation if $n$ is odd and that $\xi_{n+3 j, g-j}^{[n / 2]+j}-\beta \xi_{n+3 j, g-j-1}^{[n / 2]+j}$ is a relation if $n$ is even. Since the leading term with respect to $\alpha$ of $\xi_{r, s}^{k}$ is $\left(\begin{array}{c}r-2 k+s \\ r-2 k\end{array}\right) \alpha^{k} / k$ !, the monomial $\alpha^{n+3 j} \beta^{g-j}$ appears in these relations with a nonzero coefficient. On the other hand, by (9.1) these relations can be expressed as a linear combination of $\rho_{r, s, t}^{c} \in I_{n+2}^{g}$. Since $\rho_{n+3 j, g-j}^{g}$ is the class of maximal degree $n+3 j$ with respect to $\alpha$ among these, its coefficient in this combination must be nonzero. It is therefore indeed a relation. This completes the proof.

(12.2) Every relation between $\alpha, \beta, \gamma$ on $\mathcal{M}_{n}^{g}$ is an element of $I_{n}^{g}$.

Proof. Since the converse has just been shown, it suffices to show that $\operatorname{dim} H^{I}\left(\mathcal{M}_{n}^{g}\right)$ $=\operatorname{dim} \mathbb{Q}[\alpha, \beta, \gamma] / I_{n}^{g}$, where $H^{I}$ denotes the part of $H^{*}$ invariant under the action of both $\Sigma$ and $\Gamma$.

(In the proofs of the analogous statement for $\mathcal{N}^{g}$ [4, 16, 25, 33, it has been customary to show that all the Betti numbers match up. This can certainly be done for $\mathcal{M}_{n}^{g}$, but the cruder statement about overall dimension is clearly sufficient.)

Now since by 3.1 (i) the $\mathbb{C}^{\times}$action is perfect, the dimension of $H^{I}\left(\mathcal{M}_{n}^{g}\right)$ may be expressed as a sum over the fixed components enumerated in (4.1):

$$
\operatorname{dim} H^{I}\left(\mathcal{M}_{n}^{g}\right)=\operatorname{dim} H^{I}\left(\mathcal{N}^{g}\right)+\sum_{d=1}^{g+\left[\frac{n-1}{2}\right]} \operatorname{dim} H^{I}\left(C_{2 g+n-1-2 d}\right) .
$$

The Poincaré polynomial of $H^{I}\left(\mathcal{N}^{g}\right)$ is

$$
\frac{\left(1-t^{2 g}\right)\left(1-t^{2 g+2}\right)\left(1-t^{2 g+4}\right)}{\left(1-t^{2}\right)\left(1-t^{4}\right)\left(1-t^{6}\right)}
$$

this is clear, for example, from the presentation with three generators and three relations [4, 16, 25, 33. To find $\operatorname{dim} H^{I}\left(\mathcal{N}^{g}\right)$ we want to substitute $t=1$. Of course 0 appears in the denominator, but the limit as $t \rightarrow 1$ can easily be evaluated to $\left(\begin{array}{c}g+2 \\ 3\end{array}\right)$ by substituting $t^{2}=1+\epsilon$ and then using $(1+\epsilon)^{k}=1+k \epsilon+O\left(\epsilon^{2}\right)$.

As for $\operatorname{dim} H^{I}\left(C_{m}\right)$, it follows easily from the discussion in Arbarello et al. [1] VII B] that this equals $\left[\frac{m+2}{2}\right]\left[\frac{m+3}{2}\right]$ if $m \leq 2 g-1$ and $(g+1)(m-g+1)$ if $m \geq 2 g-1$. Hence

$$
\begin{aligned}
\operatorname{dim} & H^{I}\left(\mathcal{M}_{n}^{g}\right) \\
= & \left(\begin{array}{c}
g+2 \\
3
\end{array}\right)+\sum_{d=1}^{\left[\frac{n-1}{2}\right]}(g+1)(g-2 d+n) \\
& +\sum_{d=\left[\frac{n-1}{2}\right]+1}^{\left[\frac{n-1}{2}\right]+g}\left(g-d+1+\left[\frac{n-1}{2}\right]\right)\left(g-d+1+\left[\frac{n}{2}\right]\right) .
\end{aligned}
$$

On the other hand,

$$
\operatorname{dim} \mathbb{Q}[\alpha, \beta, \gamma] / I_{n}^{g}=\sum_{r, s, t} 1
$$


where the right-hand sum runs over all nonnegative $r, s, t$ such that $t \leq g$ and $r+3 s+3 t \leq 3 g-3+n$ or $r+2 s+2 t<2 g-2+n$. This can be rewritten as

$$
\begin{aligned}
& \sum_{t=0}^{g} \sum_{s=0}^{g-1+\left[\frac{n}{2}\right]-t} \sum_{r=0}^{\max (3 g-3+n-3 s-3 t,}{ }^{2 g-3+n-2 s-2 t)} 1 \\
& =\sum_{t=0}^{g}\left(\sum_{s=0}^{g-1-t}(3 g-2+n-3 s-3 t)+\sum_{s=0}^{g-1+\left[\frac{n}{2}\right]-t}(2 g-2+n-2 s-2 t)\right) .
\end{aligned}
$$

It is straightforward, using high-school algebra and the identities $\sum_{d=1}^{k} d=k^{2} / 2+$ $k / 2$ and $\sum_{d=1}^{k} d^{2}=k^{3} / 3+k^{2} / 2+k / 6$, to show that this equals the above formula for $\operatorname{dim} H^{I}\left(\mathcal{M}_{n}^{g}\right)$.

Proof of 2.5). The two results above show that the ideal $I\left(\mathcal{M}_{n}^{g}\right)$ of $\Gamma$-invariant relations on $\mathcal{M}_{n}^{g}$ is precisely $I_{n}^{g}$. Now apply (11.3).

\section{RELATIONSHIP With OTHER PAPERS}

The present paper is closely related to several other works by the authors; in this final section we indicate briefly a few of the points of contact.

The first author has constructed a compactification $\overline{\mathcal{H}}_{n}$ of the moduli space of Higgs bundles [1] by adding a divisor $Z_{n}$ at infinity which is a quotient by $T=\mathbb{C}^{\times}$ of an open subset of $\mathcal{H}_{n}$. Indeed, $\overline{\mathcal{H}}_{n}$ itself is a quotient by $T$ of $\mathcal{H}_{n} \times \mathbb{C}$. Many of the constructions given herein apply to $\overline{\mathcal{H}}_{n}$ and $Z_{n}$. In particular, there are direct limits $\overline{\mathcal{H}}_{\infty}$ and $Z_{\infty}$. Just as $\mathcal{H}_{\infty}$ is shown in (9.7) of our previous paper [14 to be homotopy equivalent to $B \overline{\mathcal{G}}$, we expect $\overline{\mathcal{H}}_{\infty}$, and also $Z_{\infty}$, to be homotopy equivalent to $B \mathcal{G}$, the classifying space of the full gauge group.

The cohomology rings of $\overline{\mathcal{H}}_{\infty}$ and $Z_{\infty}$, and hence those of $\overline{\mathcal{H}}_{n}$ and $Z_{n}$, will have generators like those of $\mathcal{H}_{n}$, but with one additional generator $h \in H^{2}$, corresponding to the class discarded in the proof of (10.1) of our previous paper. Indeed, the quotient map $H_{T}^{*}\left(\mathcal{H}_{n}\right) \rightarrow H^{*}\left(Z_{n}\right)$ is surjective, and $h$ is the image of $u$. It can be shown that the kernel is the image in $H_{T}^{*}\left(\mathcal{H}_{n}\right)$ of the compactly supported cohomology. The relations between the generators in $H^{*}\left(Z_{n}\right)$ are therefore of two types: those coming from relations in $H_{T}^{*}\left(\mathcal{H}_{n}\right)$ and those coming from the compactly supported cohomology. The former are covered by the results of this paper. As for the latter, they ought to be determined by the results of another paper of the first author [12, in which the intersection pairings between the cohomology and the compactly supported cohomology of $\mathcal{M}_{0}$ were computed (and shown to vanish).

By studying the stratification of $\mathcal{H}_{\infty}$ by the Harder-Narasimhan type of the underlying bundle $E$ - not $(E, \phi)$ - in the rank 2 case, the first author has been able to extract the relations in the cohomology of the lowest stratum, which retracts onto $\mathcal{N}$. This recovers the description of the ring $H^{*}(\mathcal{N})$, given by several authors [4, 16, 25, 33], in an especially simple and geometrical fashion. It will be described in a forthcoming paper [13], where it will also be shown how consideration of the Mumford conjecture leads to a natural geometrical proof of the generation theorem for the moduli space of Higgs bundles in ranks 2 and 3.

The second author has studied moduli problems providing smooth resolutions of the upward and downward flows from the components of the fixed-point set of $\mathcal{H}_{n}$ 
in the rank 2 case. The downward flow is intriguing because it can be interpreted as a master space of Bradlow pairs, but the upward flow is particularly related to the present paper. It is relatively simple to describe, but it contains the parts of $H^{*}\left(\mathcal{H}_{n}\right)$ not invariant under $\Sigma=\mathbb{Z}_{2}^{2 g}$, in the sense that none of them are killed by the restriction map. These upward flows can therefore be used to complete the description of the cohomology rings $H^{*}\left(\mathcal{H}_{n}\right)$, by characterizing the part not invariant under $\Sigma$. In fact this is not so difficult, since for dimension reasons these classes have square 0 and are killed by the $\psi_{j}$. So the products with $\alpha$ and $\beta$ are all that must be computed. Details will appear in a forthcoming paper [32].

\section{ACKNOWLEDGEMENTS}

We are grateful to the Mathematisches Forschungsinstitut Oberwolfach for its kind hospitality during three weeks in the summer of 1998, when the ideas for the present paper took shape. Computer calculations performed there in the software package Macaulay 2, by Daniel Grayson and Michael Stillman, were indispensable in formulating the main result. So were subsequent calculations in both Macaulay and Maple. We are also very grateful to Andrew Kresch for directing us to the work of Shalosh B. Ekhad, and to Ekhad for pointing out the recurrence relations that enabled us to solve the binomial coefficient identities of 9 . We thank Don Zagier for kindly sending us the proof of a delightful generating function formula from his paper [33, which became (9.2). We both wish to thank Nigel Hitchin for his advice and encouragement and Tara Brendle and Peter Newstead for helpful discussions. Finally, the first author thanks the Institute for Advanced Study and the Miller Institute for Basic Research in Science for their hospitality and support during the academic years 1998-1999 and 1999-2000, respectively.

\section{REFERENCES}

[1] E. Arbarello, M. Cornalba, P.A. Griffiths, and J. Harris, Geometry of algebraic curves, volume I, Springer-Verlag, 1985. MR 86h:14019

[2] M.F. АтіYAн and R. Bott, The Yang-Mills equations over Riemann surfaces, Philos. Trans. Roy. Soc. London Ser. A 308 (1982) 523-615. MR 85k:14006

[3] M.F. АтіYAн and R. Botт, The moment map and equivariant cohomology, Topology 23 (1984) 1-28. MR 85e:58041

[4] V. YU. Baranovskil̆, The cohomology ring of the moduli space of stable bundles with odd determinant, Izv. Ross. Akad. Nauk Ser. Mat. 58 (1994) 204-210; translation in Russian Acad. Sci. Izv. Math. 45 (1995) 207-213. MR 96a:14015

[5] K. Corlette, Flat G-bundles with canonical metrics, J. Differential Geom. 28 (1988) 361382. MR 89k:58066

[6] S.K. Donaldson, Twisted harmonic maps and the self-duality equations, Proc. London Math. Soc. (3) 55 (1987) 127-131. MR 88g:58040

[7] S.B. EkHAD, A proof of a recurrence, EKHAD, available from http://www.math.temple. edu/ zeilberg/programs.html/.

[8] W.M. Goldman, The symplectic nature of fundamental groups of surfaces, Adv. Math. 54 (1984) 200-225. MR 86i:32042

[9] D. Grayson and M. Stillman, Macaulay 2, available from http://www.math.uiuc.edu/ Macaulay2/.

[10] R.C. Gunning, Lectures on vector bundles over Riemann surfaces, Princeton, 1967. MR 37:5888

[11] T. Hausel, Compactification of moduli of Higgs bundles, J. Reine Angew. Math. 503 (1998) 169-192. MR 2000b:14036

[12] T. Hausel, Vanishing of intersection numbers on the moduli space of Higgs bundles, Adv. Theor. Math. Phys. 2 (1998) 1011-1040. MR 2000g:14017 
[13] T. HAusel, Geometric proof of the Mumford conjecture, in preparation.

[14] T. HAusel and M. Thaddeus, On the generators of the cohomology ring of the moduli space of rank 2 Higgs bundles, preprint math.AG/0003093.

[15] N.J. Hitchin, The self-duality equations on a Riemann surface, Proc. London Math. Soc. (3) 55 (1987) 59-126. MR 89a:32021

[16] A.D. King and P.E. Newstead, On the cohomology ring of the moduli space of rank 2 vector bundles on a curve, Topology 37 (1998) 407-418. MR 99a:14019

[17] F.C. KIRWAN, Cohomology of quotients in algebraic and symplectic geometry, Math. Notes 31, Princeton, 1984. MR 86i:58050

[18] F.C. KIRWAN, The cohomology rings of moduli spaces of bundles over Riemann surfaces, $J$. Amer. Math. Soc. 5 (1992) 853-906. MR 93g:14016

[19] W. Magnus, A. Karrass, and D. Solitar, Combinatorial group theory, Interscience, 1966. MR 34:7617

[20] I.G. Macdonald, Symmetric products of an algebraic curve, Topology 1 (1962) 319-343. MR 27:1445

[21] E. Markman, Generators of the cohomology ring of moduli spaces of sheaves on symplectic surfaces, preprint math.AG/0009109.

[22] V. MuÑoz, Ring structure of the Floer cohomology of $\Sigma \times S^{1}$, Topology 38 (1999) 517-528. MR 99m:57028

[23] P.E. Newstead, Characteristic classes of stable bundles over an algebraic curve, Trans. Amer. Math. Soc. 169 (1972) 337-345. MR 47:4999

[24] N. Nitsure, Moduli space of semistable pairs on a curve, Proc. London Math. Soc. (3) 62 (1991) 275-300. MR 92a:14010

[25] B. Siebert and G. Tian, Recursive relations for the cohomology ring of moduli spaces of stable bundles, Turkish J. Math. 19 (1995) 131-144. MR 96h:14013

[26] C.T. Simpson, Higgs bundles and local systems, Inst. Hautes Etudes Sci. Publ. Math. 75 (1992) 5-95. MR 94d:32027

[27] C.T. Simpson, Moduli of representations of the fundamental group of a smooth projective variety, I, Inst. Hautes Etudes Sci. Publ. Math. 79 (1994) 47-129. MR 96e:14012

[28] C.T. Simpson, Moduli of representations of the fundamental group of a smooth projective variety, II, Inst. Hautes Etudes Sci. Publ. Math. 80 (1995) 5-79. MR 96e:14013

[29] M. Thaddeus, Conformal field theory and the cohomology of the moduli space of stable bundles, J. Differential Geom. 35 (1992) 131-149. MR 93g:14017

[30] M. Thaddeus, Stable pairs, linear systems and the Verlinde formula, Invent. Math. 117 (1994) 317-353. MR 95e:14006

[31] M. Thaddeus, An introduction to the topology of the moduli space of stable bundles on a Riemann surface, Geometry and physics (Aarhus, 1995), 71-99, Lect. Notes in Pure and Appl. Math. 184, Dekker, 1997. MR 98b:14010

[32] M. Thaddeus, On the Morse decomposition of the space of Higgs bundles on a curve, in preparation.

[33] D. ZAGIER, On the cohomology of moduli spaces of rank two vector bundles over curves, The moduli space of curves (Texel Island, 1994), 533-563, Progr. Math., 129, Birkhäuser, 1995. MR 97g:14010

Department of Mathematics, University of California, Berkeley, California 94720

Current address: Department of Mathematics, University of Texas, RLM 11.168, 26th and Speedway, Austin, Texas 78712

E-mail address: hausel@math.utexas.edu

Department of Mathematics, Columbia University, 2990 Broadway, New York, New YORK 10027

E-mail address: thaddeus@math.columbia.edu 